\title{
The inner equation for one and a half degrees of freedom rapidly forced Hamiltonian systems
}

\author{
I. Baldomá \\ Dept. d'Enginyeria Informàtica i Matemàtiques. \\ Campus Sescelades Avinguda dels Països Catalans, 2643007 Tarragona, SPAIN \\ E-mail: inma.baldoma@urv.net
}

\begin{abstract}
We consider families of one and a half degrees of freedom rapidly forced Hamiltonian system which are perturbations of one degree of freedom Hamiltonians having a homoclinic connection. We derive the inner equation for this class of Hamiltonian system which is expressed as the Hamiltonian-Jacobi equation of one a half degrees of freedom Hamiltonian. The inner equation depends on a parameter not necessarily small.

We prove the existence of special solutions of the inner equation with a given behavior at infinity. We also compute the asymptotic expression for the difference between these solutions. In some perturbative cases, this asymptotic expression is strongly related with the Melnikov function associated to our initial Hamiltonian.
\end{abstract}

AMS classification scheme numbers: 37J45, 37G20, 35B40, 34E10, 34M99

Submitted to: Nonlinearity 


\section{Introduction}

The phenomenon known as splitting of separatrices has been widely studied by several authors. This phenomenon arises, for instance, when we consider a differential equation in $\mathbb{R}^{2}$ with a fixed point having coincident branches of stable and unstable manifolds and we perturb it by a periodic or quasi periodic function on time.

The simplest framework - the regular case - is when the perturbation is regular with respect to the perturbation parameter, $\varepsilon$. In such a situation, Melnikov [18] (developing some ideas by Poincaré) gave a tool, which is named Poincaré-Melnikov function, to provide asymptotic expressions of the distance (and other related quantities) between the perturbed invariant manifolds when $\varepsilon \rightarrow 0$.

If the perturbation is not regular on $\varepsilon$, for instance because it depends periodically on $t / \varepsilon$, then the Poincaré-Melnikov function does not give, a priori, the right estimate of the measure of the splitting of separatrices, which in the Hamiltonian case is always exponentially small in $\varepsilon$, (see [10] for the periodic case). These singular cases are also known as rapidly forced systems. Exponentially small splitting of separatrices phenomenon was already discovered by Poincaré [21] in a near integrable case.

In 1964, Arnold [2], when studying the diffusion on the action variables of the near integrable systems $h_{0}(I)+\varepsilon h_{1}(\varphi, I, \varepsilon)$, realized that the splitting of separatrices associated to partially hyperbolic tori was exponentially small in $\varepsilon$.

In the setting of planar systems with high frequency periodic perturbations, upper bounds of the splitting of separatrices have been given in [10], [11] and [16]. If we restrict ourselves to one and a half degrees of freedom rapidly forced Hamiltonian systems, under suitable conditions, asymptotic expressions validating the prediction given by the Poincaré-Melnikov function can be found in [7], [8], [13] and [3] (see also references therein). Two of the more important techniques used in these studies are suitable flow box coordinates around the stable invariant manifold and Extension Lemma. In [25] a more general perturbation of the pendulum is considered. The author uses a different method, based on a continuous averaging procedure, for proving an asymptotic formula of the splitting of separatrices which differs from the one predicted by the PoincaréMelnikov function.

In the examples above the given asymptotic expressions are of the form $\varepsilon^{r} \mathrm{e}^{-a / \varepsilon}$, but it is possible to find systems where the true asymptotic formula does not have this form (see [24]).

This problem can also be studied for planar maps. Lazutkin wrote the first study of this subject, [17], in which he gave an asymptotic formula for the splitting of separatrices of the standard map. The complete proof of it can be found in [14]. In this context in [12] exponentially small upper bounds for the splitting of separatrices are proved for analytic families of diffeomorphisms close to the identity. In [6], is proved an asymptotic expression for the splitting of separatrices for some perturbations of the McMillan map, which is also exponentially small and, in fact, coincides with the prediction given by the Poincaré-Melnikov function. 
In [23], the author introduces a new method to study the splitting of separatrices in Hamiltonian systems which is illustrated in the Generalized Arnol'd Model with $d+1$ degrees of freedom $(d \geq 2)$. In the model considered in [23] a fixed torus with stable and unstable invariant manifolds is left invariant after perturbation. The stable and unstable invariant manifolds are given as solutions of the Hamilton-Jacobi equation. The main tool to study the splitting of separatrices is a characteristic vector field, which is defined on a part of the configuration space, has constant coefficients in good variables and acts on the difference of the stable and unstable manifold by zero. Actually upper bounds of the splitting of separatrices are given in a general setting and also lower bounds for special cases are proved.

Recently, resurgence theory (see [9], [5]) has also been used in the problem of the exponentially small splitting of separatrices. In [22] the author studies the rapidly forced pendulum by using parametric resurgence. Resurgence theory can also be used in the study of the exponentially small splitting of separatrices for a map, see [15] where the authors deal with the Hénon map.

The study we present in this paper is close to another strategy based on matching complex techniques (see [4]). This method will allow us to study the splitting of separatrices in the singular case, for instance in the case of one and a half degrees of freedom rapidly forced Hamiltonian, $H_{\mu, \varepsilon}$ of the form

$$
H_{\mu, \varepsilon}(x, y, t / \varepsilon)=h_{0}(x, y)+\mu h_{1}(x, y, t / \varepsilon, \mu, \varepsilon)
$$

where $h_{1}$ is $2 \pi$-periodic with respect to $t / \varepsilon$. Suppose that $H_{0, \varepsilon}$ has a homoclinic connection and that it can be parameterized by a complex parameter $u \in\{z \in \mathbb{C}$ : $|\operatorname{Im} z|<a\}$ for some $a>0$. Assume that this parametrization has only two singularities on $\{z \in \mathbb{C}:|\operatorname{Im} z|=a\}$ located at points $u= \pm \mathrm{i} a$. These hypotheses are satisfied, for instance, by the pendulum. Roughly speaking the method is the following:

1) To simplify the exposition, we consider the Hamilton-Jacobi equation associated to (1.1). The perturbed invariant manifolds will be described by means of two special solutions of the Hamilton-Jacobi equation, $\phi^{ \pm}$, satisfying an asymptotic condition.

2) We prove the existence of parameterizations, $\phi^{ \pm}$, of the perturbed invariant manifolds in the so called outer domain, $O$. In this domain, the invariant manifolds $\phi^{ \pm}$are well approximated by the homoclinic connection.

3) We look for good approximations of the perturbed invariant manifolds near the singularities of the homoclinic connection. For this, we derive the inner equation, which is independent of $\varepsilon$. These approximations are merely special solutions, $\phi_{i n}^{ \pm}$, of the so called inner equation and they are useful only in a small neighborhood of the singularities: the inner domain, I. In the inner domain the homoclinic connection is not a good approximation of the invariant manifolds. It is necessary that $I \cap O \neq \emptyset$ and $O \cup I=\{z \in \mathbb{C}:|\operatorname{Im} z|<a\}$.

We also compute the asymptotic expression of the difference between $\phi_{i n}^{+}$and $\phi_{i n}^{-}$.

4) By using matching complex techniques, the functions $\phi_{i n}^{ \pm}$in the inner domain must be connected with the invariant manifolds $\phi^{ \pm}$in the outer domain. 
5) Finally, it is necessary to prove that the dominant term of the splitting of separatrices, $\phi^{-}-\phi^{+}$, is given by the one obtained in the inner domain, $\phi_{i n}^{-}-\phi_{i n}^{+}$.

By using this strategy it seems possible to deal with larger perturbations. See [19] for a good summary.

In this work we perform step 3) mentioned above by considering an inner equation which comes from a quite general one and a half degrees of freedom rapidly forced Hamiltonian. In [20], an inner equation derived from an example of a rapidly forced pendulum is studied by using equational resurgence. Note that we will not use resurgence theory: our approach is closely to the one given in [23].

Using the results of this paper, we plan, in a forthcoming work, to give an asymptotic expression for the splitting of separatrices for one and a half degrees of freedom rapidly forced Hamiltonian systems having more general perturbations than the ones considered until now.

The paper is organized as follows. In Section 2 we explain the problem and the motivation to studying it. In Section 3 we introduce notation and state the main results. Sections 4, 5 and 6 are devoted to the proof of the results of Section 3. Finally, even when the proofs we will present in this work deal with the analytic case, we have included an appendix where we state and prove similar results to the ones given in Section 3 for Hamiltonians which are only differentiable with respect to time. We distinguish between the analytic and the non-analytic dependence on time in order to clarify the exposition.

\section{Context and motivation}

\subsection{The problem}

Consider the Hamiltonian $\mathcal{H}=\mathcal{H}_{0}+\mu \mathcal{H}_{1}$, where $\mu$ is a not necessarily small parameter,

$\mathcal{H}_{0}(z, w)=\frac{1}{2} w^{2} z^{2 r}-\frac{1}{2 z^{2 r}}, \quad \mathcal{H}_{1}(z, w, \tau, \mu)=\frac{1}{z^{\ell}} \sum_{j=0}^{N} A_{j}(\tau, \mu) w^{j} z^{2 r j}$,

$r \geq 1, \ell \in \mathbb{R}, N \in \mathbb{N}$. Moreover $\left\{A_{j}\right\}_{j \in\{0, \cdots, N\}}$ are arbitrary analytic functions in $(\tau, \mu)$, $2 \pi$-periodic and having zero mean with respect to $\tau$.

Our goal is to study the existence and properties of two special solutions $\phi_{i n}^{ \pm}$of the Hamilton-Jacobi equation associated to the Hamiltonian $\mathcal{H}$ :

$$
\partial_{\tau} \phi+\mathcal{H}\left(z, \partial_{z} \phi, \tau, \mu\right)=0
$$

satisfying that $\phi_{\text {in }}^{ \pm}$are analytic in some complex domain $E^{ \pm}, \phi_{\text {in }}^{ \pm}$are $2 \pi$-periodic with respect to $\tau$ and have the asymptotic property:

$$
\lim _{z \rightarrow \pm \infty} \partial_{z} \phi_{i n}^{ \pm}(z, \tau, \mu)=0 .
$$

We are also interested in computing the asymptotic expression of the difference $\partial_{z}\left(\phi_{i n}^{-}-\phi_{i n}^{+}\right)(z, \tau, \mu)$ as $\mu \rightarrow 0$ and $\operatorname{Im} z \rightarrow-\infty$.

To shorten the notation, along this work we will denote $\phi_{i n}^{ \pm}$simply by $\phi^{ \pm}$. 


\subsection{The model: a inner equation}

The Hamiltonian defined by $\mathcal{H}$ arises naturally from Hamiltonians of the form

$$
H_{\mu, \varepsilon}(q, p, t / \varepsilon)=\frac{1}{2} p^{2}+V(q)+\mu \varepsilon^{m} h_{1}(q, p, t / \varepsilon, \mu, \varepsilon)
$$

such that the unperturbed system (given by $H_{0, \varepsilon}$ ) has a homoclinic connection. Indeed, assume that $V$ is an analytic function, $h_{1}$ is analytic with respect to $(p, q, \mu)$ and $2 \pi \varepsilon$-periodic with respect to $t$. Moreover assume that the Hamiltonian system given by $H_{\mu, \varepsilon}$, when $\mu=0$ has the origin as a saddle fixed point, that one branch of the stable invariant manifold coincides with one branch of the unstable one, given rise to a homoclinic connection, which can be parameterized by a complex parameter $u$. We denote it by $\gamma_{0}(u)=\left(q_{0}(u), p_{0}(u)\right)$ and we suppose that $\gamma_{0}(u)$ is analytic in the complex strip $S_{a}=\{u \in \mathbb{C}:|\operatorname{Im} u|<a\}$, that it has singularities at $u= \pm \mathrm{i} a$, that it has no other singularities in $\{u \in \mathbb{C}:|\operatorname{Im} u|=a\}$ and that in a neighborhood of $\pm \mathrm{i} a$, there exist $r>1, C_{ \pm} \in \mathbb{C}$ and functions $g, h$ with $g(0)=h(0)=0$ in such a way that $\gamma_{0}$ can be written as

$$
q_{0}(u)=-\frac{C_{ \pm}}{(r-1)} \frac{1}{(u \mp \mathrm{i} a)^{r-1}}(1+g(u \mp \mathrm{i} a)), \quad p_{0}(u)=\frac{C_{ \pm}}{(u \mp \mathrm{i} a)^{r}}(1+h(u \mp \mathrm{i} a)) .
$$

Without loss of generality we can assume that $V(0)=0$. Hence

$$
V\left(q_{0}(u)\right)=-\frac{p_{0}^{2}}{2}=-\frac{C_{ \pm}^{2}}{(u \mp \mathrm{i} a)^{2 r}}(1+f(u \mp \mathrm{i} a))
$$

with $f(0)=0$.

We now consider the symplectic change of variables given by

$$
\tau=\frac{t}{\varepsilon}, \quad q=q_{0}(u), \quad p=\frac{v}{p_{0}(u)},
$$

which is well defined, in a neighborhood of i $a$ intersected with $S_{a}$. We notice that the homoclinic orbit can be expressed locally in the new variables as $\left(u, p_{0}^{2}(u)\right)$ and the new Hamiltonian is merely $\bar{H}_{\mu, \varepsilon}(u, v, \tau)=\varepsilon H_{\mu, \varepsilon}\left(q_{0}(u), v / p_{0}(u), \tau\right)$. With this change of variables we have control about the definition domain of the variable $u$ (which will be a neighborhood of $\pm \mathrm{i} a$ intersected with the complex strip $S_{a}$ ). Moreover, since in these variables the homoclinic connection $\gamma_{0}$ can be written as the graph of a suitable function, we expect that also the invariant manifolds of the new Hamiltonian will be expressed as the graph of adequate functions.

We are looking for a new Hamiltonian $\mathcal{H}$ which will be a good approximation of $H_{\mu, \varepsilon}$ in a neighborhood of the singularity $u=\mathrm{i} a$ (one can proceed in an analogous way to study the singularity $u=-\mathrm{i} a$ ). For this reason, we perform the change of variables given by $z=(u-\mathrm{i} a) / \varepsilon, w=\varepsilon^{2 r} C_{+}^{-2} v$. This change has constant determinant and therefore the new system is also hamiltonian with Hamiltonian

$$
\begin{aligned}
\overline{\mathcal{H}}_{\mu, \varepsilon}(z, w, \tau)= & \varepsilon^{2 r-1} C_{+}^{-2} \bar{H}_{\mu, \varepsilon}\left(\varepsilon z+\mathrm{i} a, w \varepsilon^{-2 r} C_{+}^{2}, \tau\right) \\
= & \varepsilon^{2 r} C_{+}^{-2}\left(\frac{C_{+}^{4} w^{2}}{\varepsilon^{4 r} p_{0}^{2}(\varepsilon z+\mathrm{i} a)}+V\left(q_{0}(\varepsilon z+\mathrm{i} a)\right)\right) \\
& +C_{+}^{-2} \mu \varepsilon^{m-2 r} h_{1}\left(q_{0}(\varepsilon z+\mathrm{i} a), \frac{C_{+}^{2} w}{\varepsilon^{2 r} p_{0}(\varepsilon z+\mathrm{i} a)}, \tau, \mu, \varepsilon\right) .
\end{aligned}
$$


We assume another condition over $h_{1}: h_{1}$ is a polynomial in the $(q, p)$ variables, that is, $h_{1}(q, p, \tau, \mu, \varepsilon)=\sum_{0 \leq i, j \leq M} a_{i, j}(\tau, \mu, \varepsilon) q^{i} p^{j}$. Therefore we can define

$$
\begin{gathered}
\ell=\max \left\{(r-1) i+r j: \forall \mu_{0}, \varepsilon_{0}>0, \exists(\tau, \mu, \varepsilon) \in[0,2 \pi] \times\left[-\mu_{0}, \mu_{0}\right] \times\left(0, \varepsilon_{0}\right)\right. \\
\text { s.t. } \left.a_{i, j}(\tau, \mu, \varepsilon) \neq 0\right\} .
\end{gathered}
$$

In other words, $\ell$ is the greatest order of the singularities $\pm \mathrm{i} a$ among all the monomials of $h_{1}$. This quantity $\ell$ was also defined in [8].

Using expressions (2.5) and (2.6) of $q_{0}(u), p_{0}(u)$ and $V\left(q_{0}(u)\right)$ and taking into account the definition of $\ell$, we conclude that

$$
\overline{\mathcal{H}}_{\mu, \varepsilon}(z, w, \tau)=\mathcal{H}_{0}(z, w)+\mu \varepsilon^{m-\ell+2 r} \mathcal{H}_{1}(z, w, \tau, \mu)\left(1+f_{\varepsilon}\right)+g_{\varepsilon}
$$

where $\mathcal{H}_{0}$ and $\mathcal{H}_{1}$ are of the form $(2.1)$ and $f_{0}=g_{0}=0$.

Remark 2.1 It is not difficult to see that we also obtain a system of the form (2.7) if both $V$ and $h_{1}$ are trigonometric polynomials with respect to $q$, and $h_{1}$ is a polynomial with respect to $p$. In this case we allow $r \geq 1$.

Taking $m=\ell-2 r$, and considering system (2.7) for $\varepsilon=0$, we get a Hamiltonian system with Hamiltonian $\mathcal{H}$. Hence, the study of the existence and properties of solutions $\phi^{ \pm}$of the Hamilton-Jacobi equation (2.2) is strongly related to the study of the invariant manifolds of Hamiltonian systems of the form (2.4). Obviously, if $m>\ell-2 r$ we can rename $\mu \varepsilon^{m-\ell+2 r}$ by $\mu$ and proceeding as in the case $m=\ell-2 r$. The case $m<\ell-2 r$ remains unknown.

Remark 2.2 Consider system (2.7) for $\mu=\varepsilon=0$. In this case, the approximation of the piece of the stable (or unstable) invariant manifold we are dealing with can be represented in the new variables $(z, w)$ as $\left(z, 1 / z^{2 r}\right)$.

Remark 2.3 The previous procedure is a generalization of the idea given in [20] for obtaining an inner equation for a perturbed pendulum. In our case the homoclinic connection is not a Lagrangian manifold thus we can not deal with the Hamilton-Jacobi equation from the beginning as in [20].

\section{Main results}

Before presenting the precise statement of the results, let us fix some notation.

For any $b>0$ and $\mu_{0}>0$ we introduce the complex strip $S_{b}=\{\tau \in \mathbb{C}:|\operatorname{Im} \tau|<b\}$ and the open ball $B\left(\mu_{0}\right)=\left\{\mu \in \mathbb{C}:|\mu|<\mu_{0}\right\}$.

Let $\gamma, \rho>0$. We define the complex domains

$$
\begin{gathered}
D_{\gamma, \rho}^{+}=\{z \in \mathbb{C}:|\operatorname{Im} z|>-\gamma \operatorname{Re} z+\rho\}, \quad D_{\gamma, \rho}^{-}=-D_{\gamma, \rho}^{+}, \\
E_{\gamma, \rho}=D_{\gamma, \rho}^{+} \cap D_{\gamma, \rho}^{-} \cap\{z \in \mathbb{C}: \operatorname{Im} z<0\} .
\end{gathered}
$$

To shorten the notation we write $\mathcal{D}_{\gamma, \rho, b}^{ \pm}=D_{\gamma, \rho}^{ \pm} \times S_{b} \times B\left(\mu_{0}\right)$ and $\mathcal{E}_{\gamma, \rho, b}=E_{\gamma, \rho} \times S_{b} \times B\left(\mu_{0}\right)$.

The first result is related to the existence of analytic solutions, $\phi^{ \pm}$, of the HamiltonJacobi equation (2.2), satisfying that $\lim _{\operatorname{Re} z \rightarrow \pm \infty} \partial_{z} \phi^{ \pm}=0$ and that are $2 \pi$-periodic with respect to $\tau$. 
Theorem 3.1 Consider the Hamiltonian $\mathcal{H}=\mathcal{H}_{0}+\mu \mathcal{H}_{1}$, where

$\mathcal{H}_{0}(z, w)=\frac{1}{2} w^{2} z^{2 r}-\frac{1}{2 z^{2 r}} \quad$ and $\quad \mathcal{H}_{1}(z, w, \tau, \mu)=\frac{1}{z^{\ell}} \sum_{j=0}^{N} A_{j}(\tau, \mu) w^{j} z^{2 r j}$

with $r \geq 1, \ell \in \mathbb{R}, N \in \mathbb{N}$.

Assume that $\left\{A_{j}\right\}_{j \in\{0, \cdots, N\}}$ are analytic functions on $S_{b_{0}} \times B\left(\mu_{0}\right)$ for some $b_{0}>0$ and $\mu_{0}>0$ and that they are $2 \pi$-periodic, with zero mean, with respect to $\tau$.

Then, if $\ell \geq 2 r$, for any $\gamma>0$ and $0<b<b_{0}$ there exists $\rho_{0}=\rho_{0}\left(\gamma, b, \ell, r, \mu_{0}\right)>0$, such that the Hamilton-Jacobi equation associated to $\mathcal{H}$ :

$$
\partial_{\tau} \phi+\mathcal{H}\left(z, \partial_{z} \phi, \tau, \mu\right)=0
$$

has solutions $\phi^{ \pm}: \mathcal{D}_{\gamma, \rho_{0}, b}^{ \pm} \rightarrow \mathbb{C}$ of the form

$$
\phi^{ \pm}(z, \tau, \mu)=-\frac{1}{(2 r-1) z^{2 r-1}}+\mu \phi_{1}^{ \pm}(z, \tau, \mu)+\xi^{ \pm}, \quad \xi^{ \pm} \in \mathbb{C},
$$

where $\phi_{1}^{ \pm}$are analytic functions in all their variables and $2 \pi$-periodic with respect to $\tau$. Moreover the derivatives $\partial_{z} \phi_{1}^{ \pm}$are uniquely determined by the condition:

$$
\sup _{(z, \tau, \mu) \in \mathcal{D}_{\gamma, \rho_{0}, b}^{ \pm}}\left|z^{\ell+1} \partial_{z} \phi_{1}^{ \pm}(z, \tau, \mu)\right|<+\infty .
$$

Remark 3.2 We define $\varepsilon_{\tau}=\left(b_{0}-|\operatorname{Im} \tau|\right) / 2$ and the complex set

$$
D_{\gamma}(\tau, \mu)=\left\{z \in \mathbb{C}:|\operatorname{Im} z|>-\gamma \operatorname{Re} z+\rho_{0}\left(\gamma,|\operatorname{Im} \tau|+\varepsilon_{\tau}, \ell, r,|\mu|\right)\right\} .
$$

It can be proved that the solutions of the Hamilton-Jacobi equation given in Theorem 3.1, $\phi^{ \pm}$, are analytic functions in $(z, \tau, \mu) \in D_{\gamma}(\tau, \mu) \times S_{b_{0}} \times B\left(\mu_{0}\right)$ respectively, and therefore we do not lose the analyticity domain with respect to $(\tau, \mu)$ provided $z \in D_{\gamma}(\tau, \mu)$.

The proof of Theorem 3.1 is given in Section 4 .

Let $\phi^{ \pm}$be two solutions of the Hamilton-Jacobi equation (3.3) satisfying the conclusions of Theorem 3.1. Our goal now will be to give an asymptotic expression for the difference between $\partial_{z} \phi^{-}$and $\partial_{z} \phi^{+}$as $\operatorname{Im} z \rightarrow-\infty$.

To state the next result properly we need to introduce some notation. We write

$$
Q_{j}(\tau, \mu)=\sum_{k=j}^{N}\left(\begin{array}{c}
k \\
j
\end{array}\right) A_{k}(\tau, \mu), \quad j=0, \cdots, N
$$

and we define $F_{0}$ such that $\partial_{\tau} F_{0}=Q_{0}$ and $\left\langle F_{0}\right\rangle=0$ where $\langle\cdot\rangle$ denotes, as usual, the mean with respect to $\tau$.

Theorem 3.3 Under the conditions of Theorem 3.1, there exist $\rho_{1}=\rho_{1}\left(\gamma, b, \ell, r, \mu_{0}\right) \geq$ $\rho_{0}$, an analytic function $C(\mu)$ defined on $B\left(\mu_{0}\right)$ and an analytic function $g: \mathcal{E}_{\gamma, \rho_{1}, b} \rightarrow \mathbb{C}$ such that, for any two solutions $\phi^{ \pm}$of equation (3.3) given by Theorem 3.1,

$$
\partial_{z}\left(\phi^{-}-\phi^{+}\right)(z, \tau, \mu) \sim-\mathrm{i} \mu C(\mu) \mathrm{e}^{-\mathrm{i}(z-\tau+\mu g(z, \tau, \mu))} \quad \text { as } \operatorname{Im} z \rightarrow-\infty .
$$

We also have that

$$
-\mathrm{i} C(0) \mathrm{e}^{-\mathrm{i}(z-\tau)} \sim \ell \int_{-\infty}^{+\infty} \frac{Q_{0}(\tau+t, 0)}{(z+t)^{\ell+1}} \mathrm{~d} t \quad \text { as } \operatorname{Im} z \rightarrow-\infty,
$$


Inner equation for $1 \frac{1}{2}$ degrees of freedom Hamiltonian systems

where $\left\{A_{j}\right\}_{j \in\{0, \cdots, N\}}$ are defined by (3.2).

Moreover the function $g$ satisfies that

$\sup _{(z, \tau, \mu) \in \mathcal{E}_{\gamma, \rho_{1}, b}}\left|z^{\ell-2 r} g(z, \tau, \mu)\right|<\infty \quad$ if $\ell>2 r$

$\sup _{(z, \tau, \mu) \in \mathcal{E}_{\gamma, \rho_{1}, b}}\left|(\log |z|)^{-1} g(z, \tau, \mu)\right|<\infty$ if $\ell=2 r$ and either $Q_{1} \neq 0$ or $\left\langle F_{0} \cdot Q_{2}\right\rangle \neq 0$

$\sup _{(z, \tau, \mu) \in \mathcal{E}_{\gamma, \rho_{1}, b}}|z g(z, \tau, \mu)|<\infty \quad$ if $\ell=2 r, Q_{1}=0$ and $\left\langle F_{0} \cdot Q_{2}\right\rangle=0$.

Remark 3.4 We emphasize that the function g given in Theorem 3.3 does not depend on the choice of $\phi^{ \pm}$. In fact we will see that $g$ only depends on $\partial_{z} \phi^{ \pm}$which are determined uniquely by the condition (3.4).

The proof of Theorem 3.3 will be left until Section 5. The main idea to prove this theorem is to exploit the fact that the difference $\partial_{z}\left(\phi^{-}-\phi^{+}\right)$satisfies a linear equation with suitable properties. This idea was already introduced in [23] although the way we deal with this linear equation is different.

Let us denote $a^{k}(\mu)$ the $k$-Fourier coefficient of $Q_{0}(\tau, \mu)$. The following corollary gives an explicit asymptotic formula of $\partial_{z}\left(\phi^{-}-\phi^{+}\right)$as $\mu \rightarrow 0$ and $\operatorname{Im} z \rightarrow-\infty$.

Corollary 3.5 Under the same assumptions of Theorem 3.1 and the condition $a^{1}(0) \neq$ 0 , the following asymptotic formulas hold:

i) If either $\ell>2 r$, or $\ell=2 r, Q_{1}=0$ and $\left\langle F_{0} \cdot Q_{2}\right\rangle=0$,

$\partial_{z}\left(\phi^{-}-\phi^{+}\right)(z, \tau, \mu) \sim \mu_{\mathrm{i}}^{\ell+1} \frac{2 \pi \ell}{\Gamma(\ell+1)} a^{1}(0) \mathrm{e}^{-\mathrm{i}(z-\tau)}, \quad \operatorname{Im} z \rightarrow-\infty, \mu \rightarrow 0$.

ii) If $\ell=2 r$, and either $Q_{1} \neq 0$ or $\left\langle F_{0} \cdot Q_{2}\right\rangle \neq 0$,

$\partial_{z}\left(\phi^{-}-\phi^{+}\right)(z, \tau, \mu) \sim \mu^{\ell+1} \frac{2 \pi \ell}{\Gamma(\ell+1)} a^{1}(0) \mathrm{e}^{-\mathrm{i}(z-\tau+\mu g(z, \tau, 0))}, \quad \operatorname{Im} z \rightarrow-\infty, \mu \rightarrow 0$.

We will check Corollary 3.5 in Section 6.

If there is no danger of confusion, we will omit the dependence with respect to the parameters $\mu$ and $\mu_{0}$ in the notation. Throughout the paper this dependence will be analytic.

\subsection{Remarks}

- We stress that Theorems 3.1 and 3.3 apply for not necessarily small values of $\mu$.

- Note that our results are only valid if $\ell \geq 2 r$. The case $\ell<2 r$ must be treated differently.

- Our results agree with those on the difference between $\phi^{+}$and $\phi^{-}$given in [20] for the particular case

$$
\partial_{\tau} \phi-\frac{1}{8} z^{2}\left(\partial_{z} \phi\right)^{2}+2 \frac{1}{z^{2}}(1-\mu \sin \tau)=0 .
$$

Performing the linear change $\phi=-4 \psi$ we obtain equation (3.3) for $r=1, \ell=2$ and $\mathcal{H}_{1}=z^{-2}(\sin \tau) / 2$. In this case, $N=0$ and hence $Q_{1}=0$ and $\left\langle F_{0} \cdot Q_{2}\right\rangle=0$. 
- We notice that the asymptotic expression given in Corollary 3.5 is closely related to the Melnikov function $M$ of the Hamiltonian system $H_{\mu, \varepsilon}$, defined in (2.4). In this case we are considering $\mu$ as a small parameter. In fact, assuming the hypotheses of Subsection 2.2 if either $\ell>2 r$, or $\ell=2 r, Q_{1}=0$ and $\left\langle F_{0} \cdot Q_{2}\right\rangle=0$, we have that

$$
\mu \varepsilon^{\ell} M(u, \varepsilon) \sim 2 \operatorname{Re}\left(C_{+}^{2} \partial_{z}\left(\phi^{+}-\phi^{-}\right)((u-\mathrm{i} a) / \varepsilon, 0)\right) .
$$

Indeed, let $J(q, p, \tau)=\left\{h_{0}, h_{1}\right\}(q, p, \tau)$ and let $J_{k}(q, p)$ be its $k$-Fourier coefficient. It is clear that there exist functions $h_{k}^{ \pm}$satisfying $h_{k}^{ \pm}(0)=0$ such that

$$
J_{k}\left(q_{0}(u), p_{0}(u)\right)=\frac{J_{k, 0}^{ \pm}}{(u \pm \mathrm{i} a)^{\ell+1}}\left(1+h_{k}^{ \pm}(u \pm \mathrm{i} a)\right),
$$

in a neighborhood of $u=\mp \mathrm{i} a$ respectively. We note that $J_{k, 0}^{+}=\overline{J_{k, 0}^{-}}$. In [3] and [8] (for $\ell \in \mathbb{N}$ ) it is proved that, if $J_{1,0}^{-} \neq 0$, then

$$
\varepsilon^{\ell} M(u, \varepsilon) \sim \frac{4 \pi}{\Gamma(\ell+1)} \operatorname{Re}\left(\mathrm{i}^{\ell+1} J_{1,0}^{-} \mathrm{e}^{-i u / \varepsilon}\right) \mathrm{e}^{-a / \varepsilon} .
$$

Following the changes of variables given in Subsection 2.2, tedious but easy computations show that

$$
\sum_{j=0}^{N} A_{j}(\tau, \mu)=\frac{1}{\ell C_{+}^{2}} \sum_{k \in \mathbb{Z} \backslash\{0\}} J_{k, 0}^{-} \mathrm{e}^{\mathrm{i} k \tau} .
$$

Thus $J_{1,0}^{-}=a^{1}(0) \ell C_{+}^{2}$. Finally (3.7) follows from the asymptotic expression given in $i$ ) of Corollary 3.5.

- In the case $\ell=2 r$ and either $Q_{1} \neq 0$ or $\left\langle F_{0} \cdot Q_{2}\right\rangle \neq 0$, the difference $\partial_{z}\left(\phi^{-}-\phi^{+}\right)$has an extra term given by the function $g$ which is not related (a priori) to the Melnikov function. We expect that, in the cases where $g$ be of order $\log |z|$, the Melnikov function will not measure the splitting of separatrices even when the parameter $\mu$ is small.

This case is fulfilled, for instance, if we look for the inner equation for the perturbed pendulum

$$
H_{\mu, \varepsilon}(q, p, t / \varepsilon)=\frac{1}{2} p^{2}+(1-\cos q)+\mu\left(p^{2} \cos (t / \varepsilon)+\cos q \sin (t / \varepsilon)\right) .
$$

Indeed, the homoclinic orbits are given by

$$
\left(q_{0}(u), p_{0}(u)\right)=( \pm 2 \arctan (\sinh u), \pm 2 \operatorname{sech} u) .
$$

Let us consider the + sign. The second component has poles of order 1 at $u= \pm \mathrm{i} \pi / 2+2 k \pi$. Hence, following the notation given in Section 2, one has that $r=1, a=\pi / 2$ and $C_{+}=-2 \mathrm{i}$. Writing $h_{1}(q, p, \tau, \mu, \varepsilon)=p^{2} \cos (t / \varepsilon)+\cos q \sin (t / \varepsilon)$, we obtain

$\frac{\varepsilon^{2}}{C_{+}^{2}} h_{1}\left(q_{0}(\varepsilon z+\mathrm{i} a), \frac{C_{+}^{2} w}{\varepsilon^{2} p_{0}(\varepsilon z+\mathrm{i} a)}, \tau, \mu, \varepsilon\right)=\frac{1}{z^{2}}\left(\frac{1}{2} \sin \tau+w^{2} z^{4} \cos \tau\right)(1+O(\varepsilon z))$ and consequently,

$$
\mathcal{H}_{1}(z, w, \tau, \mu)=\frac{1}{z^{2}}\left(\frac{1}{2} \sin \tau+w^{2} z^{4} \cos \tau\right)
$$


Obviously, in this case, $\ell=2 r, Q_{0}(\tau)=\frac{1}{2} \sin \tau+\cos \tau$, and hence $F_{0}(\tau)=$ $-\frac{1}{2} \cos \tau+\sin \tau, Q_{1}(\tau)=2 \cos \tau$ and $Q_{2}(\tau)=\cos \tau$. We have that $Q_{1}(\tau) \neq 0$ and $\left\langle F_{0} \cdot Q_{2}\right\rangle=-\frac{1}{4}$.

\section{Solutions of the Hamilton-Jacobi equation}

In this section we prove Theorem 3.1. To do so we look for solutions of the HamiltonJacobi equation (3.3):

$$
\partial_{\tau} \phi^{ \pm}+\mathcal{H}\left(z, \partial_{z} \phi^{ \pm}, \tau\right)=0
$$

of the form

$$
\phi^{ \pm}(z, \tau)=\phi_{0}(z)+\mu \phi_{1}^{ \pm}(z, \tau)+\xi^{ \pm}, \quad \xi^{ \pm} \in \mathbb{C} .
$$

where $\phi_{0}(z)=-1 /\left((2 r-1) z^{2 r-1}\right)$ with the condition that, given $\gamma>0,0<b<$ $b_{0}$ and $\rho$ big enough, $\phi_{1}^{ \pm}$is analytic on $\mathcal{D}_{\gamma, \rho, b}^{ \pm}, 2 \pi$-periodic with respect to $\tau$ and $\sup _{(z, \tau) \in \mathcal{D}_{\gamma, \rho, b}^{ \pm}}\left|z^{\ell+1} \partial_{z} \phi_{1}^{ \pm}(z, \tau)\right|<\infty$.

We observe that both $\pm \phi_{0}$ are solutions of (4.1) for $\mu=0$, but since, by Remark 2.2, the homoclinic orbit of $H_{0, \varepsilon}$ can be approximated by $\left(z, \partial_{z} \phi_{0}\right)$ in the $(z, w)$ variables we choose the + sign.

We split the proof of Theorem 3.1 into five steps which are developed in Subsections $4.1,4.2,4.3,4.4$ and 4.5 below.

Our strategy to prove Theorem 3.1 will be to apply a suitable version of the fixed point equation. For that first we define the Banach space we will work with. Actually, such Banach spaces are functional spaces of Fourier series having Fourier coefficients with potential decay when $|z| \rightarrow \infty$. The precise definition and properties of these Banach spaces are given in Subsection 4.1.

In Subsection 4.2 we deduce a partial differential equation for both $\varphi^{ \pm}:=\partial_{z} \phi_{1}^{ \pm}$. Such equation can be expressed in the form

$$
\partial_{\tau} \varphi^{ \pm}+\partial_{z} \varphi^{ \pm}=F\left(\varphi^{ \pm}\right)
$$

with $F$ a known analytic function.

Clearly the operator $\mathcal{L}(\psi)=\partial_{\tau} \psi+\partial_{z} \psi$ is not bijective but has left-side inverse in the Banach spaces introduced above, which are studied in Subsection 4.3. We denote them by $\mathcal{B}^{ \pm}$.

In Subsection 4.4, we prove that the fixed point equations $\psi=\mathcal{B}^{ \pm}(F(\psi))$ deduced from equation (4.3) have two solutions $\varphi^{ \pm}$(one for the + case and another one for the - case) such that $\sup _{(z, \tau) \in \mathcal{D}_{\gamma, \rho, b}^{ \pm}}\left|z^{\ell+1} \varphi^{ \pm}(z, \tau)\right|$ is bounded.

Finally it only remains to show that there exist solutions $\phi^{ \pm}$of the initial equation (4.1) such that $\partial_{z} \phi^{ \pm}=\partial_{z} \phi_{0}+\mu \varphi^{ \pm}$. This is done in Subsection 4.5.

We denote by $\langle\cdot\rangle$ the mean with respect to $\tau$. 


\subsection{The Banach spaces: Definition and properties}

This Subsection is devoted to introducing the Banach spaces we will deal with. We also state some of their useful properties.

On the one hand, we observe that all the $2 \pi$-periodic with respect to $\tau$ solutions of the unperturbed Hamilton-Jacobi equation $\partial_{\tau} \phi+\mathcal{H}_{0}\left(z, \partial_{z} \phi\right)=0$ going to 0 as $|z|$ goes to $\infty$, do not depend on $\tau$ and they satisfy that $\partial_{z} \phi(z)= \pm z^{-2 r}$. On the other hand we are looking for $2 \pi$-periodic solutions of the Hamilton-Jacobi equation (2.2); hence we will consider spaces of Fourier series with Fourier coefficients having potential decay to 0 as $|z| \rightarrow \infty$.

Now we give a precise definition of our Banach spaces.

For $\nu \in \mathbb{R}$ and $\gamma, \rho>0$, we write $\bar{D}_{\gamma, \rho}^{ \pm}=D_{\gamma, \rho}^{ \pm} \times B\left(\mu_{0}\right)$, with $D_{\gamma, \rho}^{ \pm}$defined in (3.1), and we define the functional spaces

$$
X_{ \pm}^{\nu}=\left\{h: \bar{D}_{\gamma, \rho}^{ \pm}: h \text { is analytic and } \sup _{(z, \mu) \in \bar{D}_{\gamma, \rho}^{ \pm}}\left|z^{\nu} h(z, \mu)\right|<+\infty\right\} .
$$

It is clear that $X_{ \pm}^{\nu}$ equipped with the norm

$$
\|h\|_{\nu}=\sup _{(z, \mu) \in \bar{D}_{\gamma, \rho}^{ \pm}}\left|z^{\nu} h(z, \mu)\right|
$$

is a Banach space.

Now we define the space $\mathcal{X}_{\gamma, \rho, b}^{\nu, \pm}$ of Fourier series with coefficients in $X_{ \pm}^{\nu}$. That is, a function $f: D_{\gamma, \rho}^{ \pm} \times S_{b} \times B\left(\mu_{0}\right) \rightarrow \mathbb{C}$ belongs to $\mathcal{X}_{\gamma, \rho, b}^{\nu, \pm}$ if and only if

i) $f$ is analytic on $D_{\gamma, \rho}^{ \pm} \times S_{b} \times B\left(\mu_{0}\right)$.

ii) $f$ is $2 \pi$-periodic with respect to its second variable.

iii) Let $f_{k}$ be the $k$-Fourier coefficient of $f$. We ask $f_{k}$ to satisfy:

$$
f_{k} \in X_{ \pm}^{\nu} \quad \text { and } \quad \sum_{k \in \mathbb{Z}}\left\|f_{k}\right\|_{\nu} \mathrm{e}^{|k| b}<+\infty
$$

We endow $\mathcal{X}_{\gamma, \rho, b}^{\nu, \pm}$ with the norm

$$
\|f\|_{\mathcal{X}_{\gamma, \rho, b}^{\nu, \pm}}=\sum_{k \in \mathbb{Z}}\left\|f_{k}\right\|_{\nu} \mathrm{e}^{|k| b}
$$

and it becomes a Banach space. The proof of this fact can be found in [23].

We will write $\mathcal{X}_{\gamma, \rho, b}^{\nu}=\mathcal{X}_{\gamma, \rho, b}^{\nu, \pm}$ when we will state common properties of both Banach spaces. If there is no danger of confusion about the definition domain $D_{\gamma, \rho}^{ \pm} \times S_{b} \times B\left(\mu_{0}\right)$, we will denote

$$
\|\cdot\|_{\nu, b}=\|\cdot\|_{\mathcal{X}_{\gamma, \rho, b}^{\nu}}, \quad \text { and } \quad \mathcal{X}^{\nu}=\mathcal{X}_{\gamma, \rho, b}^{\nu}
$$

Remark 4.1 We emphasize that checking that a $2 \pi$-periodic function $f$ belongs to $\mathcal{X}^{\nu}$ is equivalent to proving that it is analytic with respect to $\mu$, that the $k$-Fourier coefficient belongs to $X_{ \pm}^{\nu}$ for $k \in \mathbb{Z}$ and that $\|f\|_{\nu, b}<+\infty$. In other words the analyticity with respect to $\tau$ is an immediate consequence of $i$ i) and $i i i)$. 
Inner equation for $1 \frac{1}{2}$ degrees of freedom Hamiltonian systems

Remark 4.2 Assume that $f \in \mathcal{X}^{\nu}$. We denote its $k$-Fourier coefficient by $f_{k}$. We note that

$$
\sup _{(z, \tau, \mu) \in \mathcal{D}_{\gamma, \rho, b}^{ \pm}}\left|z^{\nu} f(z, \tau, \mu)\right| \leq \sum_{k \in \mathbb{Z}}\left|z^{\nu} f_{k}(z, \mu) \mathrm{e}^{k \tau}\right| \leq\|f\|_{\nu, b} .
$$

Conversely, if $f: D_{\gamma, \rho}^{ \pm} \times S_{b^{\prime}} \times B\left(\mu_{0}\right) \rightarrow \mathbb{C}$ is an analytic function, satisfying that $\sup _{(z, \tau, \mu) \in \mathcal{D}_{\gamma, \rho, b^{\prime}}^{ \pm}}\left|z^{\nu} f(z, \tau, \mu)\right|<+\infty$, then for all $b<b^{\prime}, f \in \mathcal{X}_{\gamma, \rho, b}^{\nu}$. This fact follows from the estimate

$$
\left\|f_{k}\right\|_{\nu} \leq \sup _{(z, \tau, \mu) \in \mathcal{D}_{\gamma, \rho, b}^{ \pm}}\left|z^{\nu} f(z, \tau, \mu)\right| \mathrm{e}^{-|k| b^{\prime \prime}}, \quad \text { for } b<b^{\prime \prime}<b^{\prime}
$$

which is obtaining by using the equality

$f_{k}(z, \mu)=\frac{1}{2 \pi} \int_{0}^{2 \pi} f(z, \tau, \mu) \mathrm{e}^{-\mathrm{i} k \tau} \mathrm{d} \tau=\mathrm{e}^{-|k| b^{\prime \prime}} \frac{1}{2 \pi} \int_{0}^{2 \pi} f\left(z, \tau \pm \mathrm{i} b^{\prime \prime}, \mu\right) \mathrm{e}^{-\mathrm{i} k \tau} \mathrm{d} \tau$,

taking the + sign if $k<0$ and if $k \geq 0$, we choose the - sign.

The next lemma provide fundamental properties of the Banach spaces $\mathcal{X}^{\nu}$.

Lemma 4.3 Let $\gamma, \rho, b>0$ and $\nu, \eta \in \mathbb{R}$.

i) If $\nu \geq \eta, \mathcal{X}^{\nu} \subset \mathcal{X}^{\eta}$. Moreover denoting $a_{\gamma}=\left(1+\gamma^{2}\right)^{-1 / 2}$

$$
\|h\|_{\eta, b} \leq\left(\rho a_{\gamma}\right)^{\eta-\nu}\|h\|_{\nu, b}, \quad \text { if } h \in \mathcal{X}^{\nu} .
$$

ii) If $h \in \mathcal{X}^{\nu}$ and $g \in \mathcal{X}^{\eta}$, the product $h g \in \mathcal{X}^{\nu+\eta}$ and

$$
\|h g\|_{\nu+\eta, b} \leq\|h\|_{\nu, b}\|g\|_{\eta, b} .
$$

iii) If $h \in \mathcal{X}_{\gamma, \rho, b}^{\nu}$, then there exists a constant $A_{\gamma, \nu}$ such that for $l \in \mathbb{N}\{0\}$ we have that

$$
\partial_{z}^{l} h \in \mathcal{X}_{2 \gamma, 4 \rho, b}^{l+\nu} \quad \text { and } \quad\left\|\partial_{z}^{l} h\right\|_{l+\nu, b} \leq l ! C_{\gamma}^{-l} A_{\gamma, \nu}\|h\|_{\mathcal{X}_{\gamma, \rho, b}^{\nu}} .
$$

Proof. We observe that, if $z \in D_{\gamma, \rho}^{ \pm}$, then $|z| \geq \rho\left(1+\gamma^{2}\right)^{-1 / 2}$ and therefore, formula (4.5) follows easily from the definition of the norms.

Now we check $i i)$. Let $h \in \mathcal{X}^{\nu}$ and $g \in \mathcal{X}^{\eta}$. We denote by $h_{k}, g_{k}$ and $(h g)_{k}$ the $k$-Fourier coefficients of $h, g$ and $h g$ respectively. It is clear that, for $z \in D_{\gamma, \rho}^{ \pm}$

$$
\left|z^{\nu+\eta}(h g)_{k}(z)\right| \leq \sum_{i \in \mathbb{Z}}\left|z^{\nu} h_{i}(z)\left\|z^{\eta} g_{k-i}(z) \mid \leq \sum_{i \in \mathbb{Z}}\right\| h_{i}\left\|_{\nu}\right\| g_{k-i} \|_{\eta}<+\infty .\right.
$$

Therefore, since $|k| \leq|k-i|+|i|$,

$$
\|h g\|_{\nu+\eta, b} \leq \sum_{k, i \in \mathbb{Z}}\left\|h_{i}\right\|_{\nu}\left\|g_{k-i}\right\|_{\eta} \mathrm{e}^{|k| b} \leq\|h\|_{\nu, b}\|g\|_{\eta, b}
$$

and we obtain (4.6).

Finally we prove $i i i)$. Let $l \in \mathbb{N} \backslash\{0\}$. It is clear that $\partial_{z}^{l} h$ satisfies conditions i) and ii) of the definition of the Banach spaces $\mathcal{X}_{2 \gamma, 4 \rho, b}^{l+\nu}$. To checking condition iii), we introduce the constant $C_{\gamma}=\gamma\left(4\left(1+\gamma^{2}\right)^{1 / 2}\left(1+4 \gamma^{2}\right)^{1 / 2}\right)^{-1}<1 / 4$. Geometric arguments allow us to deduce that

$$
\left\{u \in \mathbb{C}:|u-z| \leq C_{\gamma}|z|\right\} \subset D_{\gamma, \rho}^{ \pm}, \quad \text { if } z \in D_{2 \gamma, 4 \rho}^{ \pm} .
$$


Let $h_{k}$ be the $k$-Fourier coefficient of $h$. By Cauchy's formula,

$\left|\partial_{z}^{l} h_{k}(z)\right| \leq \frac{l !}{2 \pi|z|^{l} C_{\gamma}^{l}} \int_{0}^{2 \pi}\left|h_{k}\left(z+|z| C_{\gamma} \mathrm{e}^{\mathrm{i} \theta}\right)\right| \mathrm{d} \theta \leq \frac{\left\|h_{k}\right\|_{\nu} l !}{|z|^{l+\nu} C_{\gamma}^{l} \max \left\{\left(1+C_{\gamma}\right)^{\nu},\left(1-C_{\gamma}\right)^{\nu}\right\}}$,

for $z \in D_{2 \gamma, 4 \rho}^{ \pm}$and hence, summing the corresponding Fourier series, we deduce (4.7).

The following lemma deals with the composition of functions belonging to the spaces $\mathcal{X}^{\kappa}$ and $\mathcal{X}^{\nu}$ of the form $F(z+\mu f(z, \tau), \tau)$.

Lemma 4.4 Let $\gamma, \rho, b>0, \nu \in \mathbb{R}$ and $\kappa>-1$. We fix $F \in \mathcal{X}_{\gamma, \rho, b}^{\nu}$ and $f \in \mathcal{X}_{\gamma, \rho, b}^{\kappa}$. Assume that $\rho$ satisfies the inequality

$$
\mu_{0} C_{\gamma}^{-1}\|f\|_{\mathcal{X}_{\gamma, \rho, b}^{\kappa}} \leq \frac{1}{2}\left(\frac{\rho}{\left(1+\gamma^{2}\right)^{1 / 2}}\right)^{\kappa+1}
$$

with $C_{\gamma}$ satisfying (4.8). We define the formal expression

$$
\mathcal{T}_{F}(f)(z, \tau)=\sum_{l \geq 1} \frac{1}{l !} \partial_{z}^{l} F(z, \tau) \mu^{l} f^{l}(z, \tau) .
$$

Then there exists a constant $B_{\gamma, \nu}$ such that

$$
\mathcal{T}_{F}(f) \in \mathcal{X}_{2 \gamma, 4 \rho, b}^{\nu+\kappa+1}, \quad\left\|\mathcal{T}_{F}(f)\right\|_{\nu+\kappa+1, b} \leq \mu_{0} B_{\gamma, \nu}\|F\|_{\mathcal{X}_{\gamma, \rho, b}^{\nu}}\|f\|_{\mathcal{X}_{\gamma, \rho, b}^{\kappa}} .
$$

Moreover, the function $\tilde{F}(z, \tau)=F(z+\mu f(z, \tau), \tau)$ belongs to $\mathcal{X}_{2 \gamma, 4 \rho, b}^{\nu}$.

Proof. We fix $\gamma, b, \rho, \nu>0$ and $\kappa>-1$. From now on we will denote $\mathcal{X}_{2 \gamma, 4 \rho, b}^{\eta}$ simply by $\mathcal{X}^{\eta}$ and consequently we will write $\|\cdot\|_{\eta, b}=\|\cdot\|_{\mathcal{X}_{2 \gamma, 4 \rho, b}^{\eta}}$.

Given $F \in \mathcal{X}_{\gamma, \rho, b}^{\nu}$, by $\left.i i i\right)$ of Lemma 4.3, we get that for all $l \in \mathbb{N} \backslash\{0\}$

$$
\partial_{z}^{l} F \in \mathcal{X}^{l+\nu} \quad \text { and } \quad\left\|\partial_{z}^{l} F\right\|_{l+\nu, b} \leq l ! C_{\gamma}^{-l} A_{\gamma, \nu}\|F\|_{\mathcal{X}_{\gamma, \rho, b}^{\nu}} .
$$

Let now $f \in \mathcal{X}_{\gamma, \rho, b}^{\kappa} \subset \mathcal{X}^{\kappa}$. Since $\kappa+1>0$, by $\left.i i\right)$ Lemma 4.3 and (4.12), we have that $\partial_{z}^{l} F \cdot f^{l} \in \mathcal{X}^{l(\kappa+1)+\nu} \subset \mathcal{X}^{\kappa+1+\nu}$. Denoting $\rho_{\gamma}=\rho\left(1+\gamma^{2}\right)^{-1 / 2}$ and using again Lemma 4.3, we have that

$$
\begin{aligned}
\left\|\partial_{z}^{l} F \cdot f^{l}\right\|_{\kappa+1+\nu, b} & \leq \rho_{\gamma}^{(-l+1)(\kappa+1)}\left\|\partial_{z}^{l} F\right\|_{l+\nu, b}\|f\|_{\kappa, b}^{l} \\
& \leq l ! A_{\gamma, \nu} C_{\gamma}^{-1}\left(\rho_{\gamma}^{\kappa+1} C_{\gamma}\right)^{-l+1}\|F\|_{\mathcal{X}_{\gamma, \rho, b}^{\nu}}\|f\|_{\kappa, b}^{l} .
\end{aligned}
$$

Then we have that $\mathcal{T}_{F}(f)$ is a series of functions belonging to $\mathcal{X}^{\kappa+1+\nu}$. Moreover, since $\rho$ satisfies (4.9) and $\|f\|_{\kappa, b} \leq\|f\|_{\mathcal{X}_{\gamma, \rho, b}^{\kappa}}$, the constant $\rho_{\gamma}$ satisfies $\rho_{\gamma}^{-\kappa-1} \mu_{0} C_{\gamma}^{-1}\|f\|_{\kappa, b} \leq 1 / 2$ and therefore we have that

$$
\begin{aligned}
\left\|\mathcal{T}_{F}(f)\right\|_{\kappa+1+\nu, b} & \leq \sum_{l \geq 1} \frac{1}{l !}|\mu|^{l}\left\|\partial_{z}^{l} F \cdot f^{l}\right\|_{\kappa+1+\nu, b} \\
& \leq A_{\gamma, \nu} C_{\gamma}^{-1}\|F\|_{\mathcal{X}_{\gamma, \rho, b}^{\nu}} \sum_{l \geq 1}\left(\rho_{\gamma}^{(\kappa+1)} C_{\gamma}\right)^{-l+1} \mu_{0}^{l}\|f\|_{\kappa, b}^{l} \\
& \leq 2 A_{\gamma, \nu} C_{\gamma}^{-1} \mu_{0}\|F\|_{\mathcal{X}_{\gamma, \rho, b}^{\nu}}\|f\|_{\mathcal{X}_{\gamma, \rho, b}^{\kappa}}
\end{aligned}
$$

and (4.11) is proved.

Finally, we notice that since the condition (4.9) is fulfilled by $f$ and $\rho$, by Remark 4.2,

$$
|\mu f(z, \tau)| \leq|z|^{-\kappa} \mu_{0}\|f\|_{\nu, b} \leq|z| \rho_{\gamma}^{-\kappa-1} \mu_{0}\|f\|_{\mathcal{X}_{\gamma, \rho, b}^{\nu}}<C_{\gamma}|z| .
$$


Hence by (4.8), $z+\mu f(z, \tau) \in D_{\gamma, \rho}$ for all $(z, \tau) \in D_{2 \gamma, 4 \rho}^{ \pm} \times S_{b}$. Then it is clear that, by Taylor's theorem $\tilde{F}=F+\mathcal{T}_{F}(f)$ and therefore by (4.11), $\tilde{F} \in \mathcal{X}^{\nu}$ provided $\mathcal{T}_{F}(f) \in \mathcal{X}^{\kappa+1+\nu} \subset \mathcal{X}^{\nu}$

From now on we deal only with the + case, the - case being analogous. For this reason we will skip the + sign of our notation in the remaining part of this Section.

\subsection{The partial differential equation $\partial_{z} \phi_{1}$ satisfies}

Since $\phi_{0}$ is a solution of equation (3.3), for $\mu=0, \phi=\phi_{0}+\mu \phi_{1}$ will be solutions of the Hamilton-Jacobi equation (3.3) if and only if $\phi_{1}$ satisfy the equation:

$$
\partial_{\tau} \phi_{1}+\partial_{z} \phi_{1}+\mathcal{H}_{1}\left(z, \partial_{z} \phi_{0}+\mu \partial_{z} \phi_{1}, \tau\right)+\frac{\mu}{2} z^{2 r}\left(\partial_{z} \phi_{1}\right)^{2}=0
$$

In order to shorten the notation, we introduce

$$
\begin{array}{cl}
Q_{j}(\tau)=\sum_{k=j}^{N}\left(\begin{array}{l}
k \\
j
\end{array}\right) A_{k}(\tau), & \chi_{1}^{\ell}(z, \tau)=-\frac{\ell}{z^{\ell+1}} Q_{0}(\tau), \\
\chi_{2}^{\ell}(z, w, \tau)=\frac{1}{z^{\ell}} Q_{1}(\tau) \mu z^{2 r} w, & \chi_{3}^{\ell}(z, w, \tau)=\frac{\mu}{2} z^{2 r} w^{2}+\frac{1}{z^{\ell}} \sum_{j=2}^{N} Q_{j}(\tau)\left(\mu z^{2 r} w\right)^{j}
\end{array}
$$

and we recall that $\left\{A_{k}\right\}_{k \in\{0, \cdots, N\}}$ are determined by $\mathcal{H}_{1}(z, w, \tau)=\sum_{k=0}^{N} A_{k}(\tau) w^{k} z^{2 r k-\ell}$.

Finally, differentiating equation (4.14) with respect to $z$ and denoting $\partial_{z} \phi_{1}$ by $\varphi$, it is not difficult to check that $\varphi$ must satisfy:

$$
\partial_{\tau} \varphi+\partial_{z} \varphi+\chi_{1}^{\ell}(z, \tau)+\partial_{z}\left(\chi_{2}^{\ell}(z, \varphi, \tau)+\chi_{3}^{\ell}(z, \varphi, \tau)\right)=0 .
$$

4.2.1. The case $\ell=2 r$ We study the particular case $\ell=2 r$. Since $Q_{1}$ has zero mean, the function determined by $\partial_{\tau} F_{1}=Q_{1}$ and $\left\langle F_{1}\right\rangle=0$ is $2 \pi$-periodic. Performing the change of variables given by

$$
u=z-\mu F_{1}(\tau), \quad \widetilde{\varphi}(u, \tau)=\varphi\left(u+\mu F_{1}(\tau), \tau\right)
$$

and denoting again $\widetilde{\varphi}$ by $\varphi$ and $u$ by $z$, equation (4.16) becomes

$$
\partial_{\tau} \varphi+\partial_{z} \varphi+\chi_{1}^{\ell}(Z(z, \tau), \tau)+\partial_{z}\left(\chi_{3}^{2 r}(Z(z, \tau), \varphi, \tau)\right)=0
$$

where $Z(z, \tau)=z+\mu F_{1}(\tau)$.

4.2.2. The final equation for $\partial_{z} \phi_{1}$ in the case $\ell \geq 2 r$ To write (4.16) and (4.17) in a unified way we introduce the functions

$$
\psi_{1}^{\ell}(z, \tau)=\left\{\begin{array}{l}
-\chi_{1}^{\ell}(z, \tau) \text { if } \ell>2 r \\
-\chi_{1}^{2 r}(Z(z, \tau), \tau) \text { if } \ell=2 r
\end{array}\right.
$$

and

$$
\psi_{2}^{\ell}(\varphi)(z, \tau)=\left\{\begin{array}{l}
-\chi_{2}^{\ell}(z, \varphi, \tau)-\chi_{3}^{\ell}(z, \varphi, \tau) \text { if } \ell>2 r \\
-\chi_{3}^{2 r}(Z(z, \tau), \varphi, \tau) \text { if } \ell=2 r
\end{array}\right.
$$

With this notation, equations (4.16) and (4.17) become

$$
\partial_{\tau} \varphi+\partial_{z} \varphi=\psi_{1}^{\ell}+\partial_{z}\left(\psi_{2}^{\ell}(\varphi)\right)
$$


Inner equation for $1 \frac{1}{2}$ degrees of freedom Hamiltonian systems

\subsection{The operator $\mathcal{B}$}

In this subsection we will study the operator $\mathcal{B}$ formally defined by

$$
\mathcal{B}(h)(z, \tau)=\int_{+\infty}^{0} h(z+t, \tau+t) \mathrm{d} t .
$$

Remark 4.5 We note that, differentiating formally under the integral sign, $\partial_{\tau} \mathcal{B}(h)+$ $\partial_{z} \mathcal{B}(h)=h$. Hence the operator $\mathcal{B}$ is a (formal) left-inverse of $\mathcal{L}(\psi)=\partial_{\tau} \psi+\partial_{z} \psi$.

The next lemma ensures that the operator $\mathcal{B}$ is actually a left-inverse of $\mathcal{L}$ in $\mathcal{X}_{\gamma, \rho, b}^{\nu}$.

Lemma 4.6 Let $\rho, \gamma, b>0$ and $\nu>1$. Then

i) The operator $\mathcal{B}: \mathcal{X}^{\nu} \rightarrow \mathcal{X}^{\nu-1}$ is well defined. Moreover, there exists a constant $K_{\nu, \gamma}$ depending only on $\nu$ and $\gamma$, such that

$$
\|\mathcal{B}(h)\|_{\nu-1, b} \leq K_{\nu, \gamma}\|h\|_{\nu, b} \quad \text { if } h \in \mathcal{X}^{\nu} .
$$

ii) Let $h \in \mathcal{X}^{\nu}$. Then $\partial_{z} \mathcal{B}(h) \in \mathcal{X}^{\nu}$ and there exists a constant $C_{\nu, \gamma}$ such that

$$
\left\|\partial_{z} \mathcal{B}(h)\right\|_{\nu, b} \leq C_{\nu, \gamma}\|h\|_{\nu, b} .
$$

iii) For $h \in \mathcal{X}^{\nu}$ with $\langle h\rangle=0$, we have that $\mathcal{B}(h) \in \mathcal{X}^{\nu},\langle\mathcal{B}(h)\rangle=0$ and

$$
\|\mathcal{B}(h)\|_{\nu, b} \leq C_{\nu, \gamma}\|h\|_{\nu, b} .
$$

Proof. First we observe that if $h \in \mathcal{X}^{\nu}$, for all $(z, \tau) \in D_{\gamma, \rho} \times S_{b},|h(z, \tau)| \leq|z|^{-\nu}\|h\|_{\nu, b}$. Therefore, if $\nu>1$, using Fubbini's theorem, we can express the $k$-Fourier coefficient of $\mathcal{B}(h)$ as

$$
(\mathcal{B}(h))_{k}(z)=\int_{+\infty}^{0} \mathrm{e}^{\mathrm{i} k t} h_{k}(z+t) \mathrm{d} t
$$

where $h_{k}$ denotes the $k$-Fourier coefficient of $h$.

We fix $\rho, \gamma, b>0, \nu>1$ and $h \in \mathcal{X}^{\nu}$. First we deal with $i$ ). On the one hand,

$$
\int_{0}^{+\infty} \frac{1}{|z+t|^{\nu}} \mathrm{d} t \leq K_{\nu, \gamma} \frac{1}{|z|^{\nu-1}} \quad \text { if } z \in D_{\gamma, \rho},
$$

where $K_{\nu, \gamma}=2\left(1+\gamma^{2}\right)^{(\nu-1) / 2} \gamma^{1-\nu} \int_{0}^{+\infty}\left(1+t^{2}\right)^{-\nu / 2} \mathrm{~d} t$. Bound (4.24) is straightforward by using the fact that if $\operatorname{Re} z<0$ and $z \in D_{\gamma, \rho}, \gamma|z| \leq\left(1+\gamma^{2}\right)^{1 / 2}|\operatorname{Im} z|$. The case $\operatorname{Re} z \geq 0$ is obvious.

On the other hand, using $\left|h_{k}(z+t)\right| \leq\left\|h_{k}\right\|_{\nu}|z+t|^{-\nu}$ in (4.23) and applying bound (4.24) we deduce

$$
\left|(\mathcal{B}(h))_{k}(z)\right| \leq\left\|h_{k}\right\|_{\nu} \int_{0}^{+\infty} \frac{1}{|z+t|^{\nu}} \mathrm{d} t \leq K_{\nu, \gamma}\left\|h_{k}\right\|_{\nu} \frac{1}{|z|^{\nu-1}}, \quad \text { if } z \in D_{\gamma, \rho} .
$$

Hence $\left\|(\mathcal{B}(h))_{k}\right\|_{\nu-1} \leq K_{\nu, \gamma}\left\|h_{k}\right\|_{\nu}$ and by using the definition of $\|\cdot\|_{\nu, b}$ we conclude that $\|\mathcal{B}(h)\|_{\nu-1, b} \leq K_{\nu, \gamma}\|h\|_{\nu, b}$.

Before checking $i$ ) and $i i i)$ we claim that if $h \in \mathcal{X}^{\nu}$,

$$
\left\|(\mathcal{B}(h))_{k}\right\|_{\nu} \leq \tilde{C}_{\nu, \gamma}|k|^{-1}\left\|h_{k}\right\|_{\nu} \quad \text { for } k \in \mathbb{Z} \backslash\{0\}
$$


with $\tilde{C}_{\nu, \gamma}=(\sin \tilde{\gamma})^{-\nu-1}$ and $\tilde{\gamma}=(\arctan \gamma / 2)$. Indeed, we fix $k>0$ and $z \in D_{\gamma, \rho}$. Clearly, $z+t \mathrm{e}^{\mathrm{i} \tilde{\gamma}} \in D_{\gamma, \rho}$, for $t \geq 0$. Then, since $h_{k}$ is analytic in $D_{\gamma, \rho}$ and $\lim _{\operatorname{Re} z \rightarrow+\infty} z h_{k}(z)=0$, Cauchy's theorem implies that we can move the path of integration $z+t$ to $z+t \mathrm{e}^{\mathrm{i} \tilde{\gamma}}$ :

$\left(\mathcal{B}^{+}(h)\right)_{k}(z)=\int_{+\infty}^{0} \mathrm{e}^{\mathrm{i} k t} h_{k}(z+t) \mathrm{d} t=\int_{+\infty}^{0} \mathrm{e}^{\mathrm{i} k t \mathrm{e}^{\mathrm{i} \tilde{\gamma}}} h_{k}\left(z+t \mathrm{e}^{\mathrm{i} \tilde{\gamma}}\right) \mathrm{e}^{\mathrm{i} \tilde{\gamma}} \mathrm{d} t$.

On the other hand, using the fact that $\arg (z) \in(-\pi+\arctan \gamma, \pi-\arctan \gamma)$, it is easy to check that $\left|z+t \mathrm{e}^{\mathrm{i} \tilde{\gamma}}\right| \geq|z| \sin \tilde{\gamma}$ and therefore, bounding the last integral in (4.26),

$\left|(\mathcal{B}(h))_{k}(z)\right| \leq\left\|h_{k}\right\|_{\nu} \int_{0}^{+\infty} \frac{\mathrm{e}^{-k t \sin \tilde{\gamma}}}{\left|z+t \mathrm{e}^{\mathrm{i} \tilde{\gamma}}\right|^{\nu}} \mathrm{d} t \leq\left\|h_{k}\right\|_{\nu} \frac{1}{\mid k \|_{|z|^{\nu}(\sin \tilde{\gamma})^{\nu+1}}}$

In the same way, if $k<0$, we choose the path of integration $t=s \mathrm{e}^{-i \tilde{\gamma}}, s \geq 0$ in (4.23) and we obtain the same bound as in (4.27). This proves (4.25).

Next we prove $i i$ ). We have already seen that $\mathcal{B}(h) \in \mathcal{X}^{\nu-1}$. Therefore, integrating by parts in (4.23), we obtain an expression for the $k$-Fourier coefficient of $\partial_{z} \mathcal{B}(h)$ which is: $\partial_{z}(\mathcal{B}(h))_{k}=h_{k}-\mathrm{i} k(\mathcal{B}(h))_{k}$. Now bound (4.25) implies $\left.i i\right)$ :

$$
\left\|\partial_{z} \mathcal{B}(h)\right\|_{\nu, b} \leq\|h\|_{\nu, b}+\sum_{k \in \mathbb{Z} \backslash\{0\}}|k|\left\|(\mathcal{B}(h))_{k}\right\|_{\nu} \mathrm{e}^{|k| b} \leq\left(1+\tilde{C}_{\nu, \gamma}\right)\|h\|_{\nu, b} .
$$

To check $i i i)$ is straightforward. Indeed, by (4.23), if $h_{0}=\langle h\rangle=0$, then $\langle\mathcal{B}(h)\rangle=0$. Finally, bound (4.22) follows from (4.25).

Remark 4.7 Given $\nu>1$ and $h \in \mathcal{X}^{\nu}$, if $\psi$ is a solution of $\mathcal{L}(\psi)=h$ such that $\lim _{\operatorname{Re} z \rightarrow+\infty} \psi(z, \tau)=0$, then $\psi=\mathcal{B}(h)$.

Indeed, let $\psi$ be a solution of $\mathcal{L}(\psi)=h$. By Lemma 4.6, $\mathcal{B}(h)$ is a solution of $\mathcal{L}(\psi)=h$, thus there exists a function $\chi$ such that $\psi=\mathcal{B}(h)+\chi(z-\tau)$. Moreover, since $\psi$ and $\mathcal{B}(h)$ are $2 \pi$-periodic with respect to $\tau$ and they both satisfy $\lim _{\operatorname{Re} z \rightarrow+\infty} \psi(z, \tau)=\lim _{\operatorname{Re} z \rightarrow+\infty} \mathcal{B}(h)(z, \tau)=0$, the function $\chi$ is $2 \pi$-periodic and it also satisfies that $\lim _{\zeta \rightarrow \infty} \chi(\zeta)=0$. This implies that $\chi \equiv 0$.

\subsection{Existence, uniqueness and asymptotic properties of $\partial_{z} \phi_{1}$}

We have seen in Subsection 4.2 that $\varphi:=\partial_{z} \phi_{1}$ has to be a solution of the partial differential equation (4.16). This subsection is devoted to proving that equation (4.16) has only one solution with the properties required for $\partial_{z} \phi_{1}$. Concretely we will prove:

Proposition 4.8 Let $\gamma>0$ and $0<b<b_{0}$. If $\ell \geq 2 r$, there exists $\rho_{0}$ depending on $\gamma, b, r$ and $\ell$, such that the partial differential equation (4.16) has only one solution $\varphi$ $2 \pi$-periodic with respect to $\tau$ and with the asymptotic property $\lim _{\operatorname{Re} z \rightarrow+\infty} \varphi(z, \tau)=0$. Moreover $\varphi \in \mathcal{X}_{\gamma, \rho_{0}, b}^{\ell+1}$ and $\varphi-\mathcal{B}\left(\psi_{1}^{\ell}\right) \in \mathcal{X}^{\ell+1+\eta_{\ell}}$ with $\eta_{\ell}=\ell-2 r$ if $\ell>2 r$ and $\eta_{\ell}=1$ if $\ell=2 r$.

Let us consider equation (4.20):

$$
\partial_{\tau} \varphi+\partial_{z} \varphi=\psi_{1}^{\ell}+\partial_{z}\left(\psi_{2}^{\ell}(\varphi)\right)
$$


(we recall that $\left\{\psi_{i}^{\ell}\right\}_{i=1,2}$ were defined by (4.18) and (4.19)). We also stress that equation (4.20) was deduced from equation (4.16) simply by performing a change of variables if $\ell=2 r$. If $\ell>2 r$ both equations are the same.

We observe that equation (4.20) can be (formally) written as a fixed point equation. Indeed, we only need to take into account that the operator $\mathcal{B}$ is linear and by Remark 4.5, $\mathcal{B}=\mathcal{L}^{-1}$. Moreover $\mathcal{B} \circ \partial_{z}=\partial_{z} \circ \mathcal{B}$ (differentiating formally under the integral sign). Hence equation (4.20) can be formally expressed as:

$$
\varphi=\mathcal{B}\left(\psi_{1}^{\ell}\right)+\partial_{z} \mathcal{B}\left(\psi_{2}^{\ell}(\varphi)\right) .
$$

To prove Proposition 4.8 we perform two steps. The first is devoted to proving that equation (4.28) has a fixed point $\varphi$ belonging to $\mathcal{X}_{\gamma, \tilde{\rho}_{0}, b}^{\ell+1}$ with $\tilde{\rho}_{0}$ large enough.

Later we will check that $\varphi$ is a solution of equation (4.20) provided that (after restrict our definition domain $D_{\gamma, \tilde{\rho}_{0}, b}$ if it is necessary) the linear operators $\partial_{z}$ and $\mathcal{B}$ actually commutes. Note that this fact implies that equation (4.28) is equivalent to

$$
\varphi=\mathcal{B}\left(\psi_{1}^{\ell}+\partial_{z}\left(\psi^{\ell}(\varphi)\right)\right)
$$

and hence by Remark 4.5, $\varphi$ is a solution of equation (4.20). The uniqueness of this solution comes from Remark 4.7 .

Finally, taking into account the relation between equations (4.20) and (4.16) we will conclude that $\varphi$ is a solution of (4.16) if $\ell>2 r$. If $\ell=2 r$, we will need to perform the change of coordinates given by $\tilde{\varphi}(z, \tau)=\varphi\left(z-\mu F_{1}(\tau), \tau\right)$, with $F_{1}$ a suitable periodic function, to obtain the solution of equation (4.16) we are looking for.

4.4.1. The fixed point equation Before dealing with the fixed point equation (4.28), we state an auxiliary lemma which works in a more general setting.

Lemma 4.9 We fix $\gamma, b, \rho>0, \nu>1$ and $h_{0} \in \mathcal{X}^{\nu}$ and we define $R_{0}=8\left\|h_{0}\right\|_{\nu, b}+1 / 2$. We denote by $B(R)$ the open ball of $\mathcal{X}^{\nu}$ of radius $R>0$ and centered at the origin.

Let $\mathcal{R}$ be an analytic operator $\mathcal{R}: B\left(2 R_{0}\right) \rightarrow \mathcal{X}^{0}$ such that there exist $C, \eta>0$ satisfying

$\mathcal{R}(0)=0, \quad \partial_{h}^{j} \mathcal{R}(0) \in \mathcal{X}^{\eta-(j-1) \nu} \quad$ and $\quad\left\|\partial_{h}^{j} \mathcal{R}(0)\right\|_{\eta-(j-1) \nu, b} \leq C \frac{j !}{\left(2 R_{0}\right)^{j}}, \quad$ for $j \geq 1$.

Then there exists $\rho_{1}=\rho_{1}(\gamma, b, \nu, \eta, \rho)$ big enough such that the operator

$$
\mathcal{F}(h):=h_{0}+\partial_{z} \mathcal{B}(\mathcal{R}(h))
$$

has a fixed point $h \in \mathcal{X}_{\gamma, \rho_{1}, b}^{\nu}$.

Proof. To shorten the notation, along this proof we will denote $\mathcal{X}_{\gamma, \rho_{1}, b}^{\nu}$ and $\|\cdot\|_{\mathcal{X}_{\gamma, \rho_{1}, b}^{\nu}}$ simply by $\mathcal{X}^{\nu}$ and $\|\cdot\|_{\nu, b}$ respectively.

We take $\rho_{1}=\max \left\{\rho,\left(1+\gamma^{2}\right)^{1 / 2}\left(16 C R_{0}^{-1} C_{\nu, \gamma}\right)^{1 / \eta}\right\}$ where $C_{\nu, \gamma}$ is the constant defined in $i$ ) of Lemma 4.6. This choice will be justified below.

In [1] it was proved that if $f$ is an analytic operator defined in a complex Banach space, satisfying that $f(B(R)) \subset B(\theta R)$ for some $\theta<1 / 2$, then $f$ has a unique fixed 
point belonging to $B(\theta R)$. Since the operator $\mathcal{F}$ is analytic, we are allowed to use this result. Specifically we will see that $\mathcal{F}\left(B\left(R_{0}\right)\right) \subset B\left(R_{0} / 4\right)$.

We fix $h \in B\left(R_{0}\right)$. We claim that,

$$
\mathcal{R}(h) \in \mathcal{X}^{\nu+\eta} \quad \text { and } \quad\|\mathcal{R}(h)\|_{\nu+\eta, b} \leq C
$$

with $C$ the constant given in Lemma 4.9. Indeed, since $\mathcal{R}$ is analytic and $\mathcal{R}(0)=0$, we have that

$$
\mathcal{R}(h)=\sum_{j \geq 1} \partial_{h}^{j} \mathcal{R}(0) \frac{h^{j}}{j !} .
$$

Since $\partial_{h}^{j} \mathcal{R}(0) \in \mathcal{X}^{\eta-(j-1) \nu}$, using the fact that $h \in B\left(R_{0}\right) \subset \mathcal{X}^{\nu}$ and $\left.i i\right)$ of Lemma 4.3, we have that for all $j \geq 1, \partial_{h}^{j} \mathcal{R}(0) h^{j} \in \mathcal{X}^{\eta-(j-1) \nu+j \nu}=\mathcal{X}^{\eta+\nu}$ and moreover, taking into account that $\|h\|_{\nu, b} \leq R_{0}$ and $\left\|\partial_{h}^{j} \mathcal{R}(0)\right\|_{\eta-(j-1) \nu, b} \leq\left\|\partial_{h}^{j} \mathcal{R}(0)\right\|_{\mathcal{X}_{\gamma, \rho, b}^{\eta-(j-1) \nu}} \leq C j !\left(2 R_{0}\right)^{-j}$,

$$
\left\|\partial_{h}^{j} \mathcal{R}(0) h^{j}\right\|_{\eta+\nu, b} \leq\left\|\partial_{h}^{j} \mathcal{R}(0)\right\|_{\eta-(j-1) \nu, b}\|h\|_{\nu, b}^{j} \leq C j ! \frac{1}{2^{j}} .
$$

Hence, $\mathcal{R}(h) \in \mathcal{X}^{\nu+\eta}$ and

$$
\|\mathcal{R}(h)\|_{\nu+\eta, b} \leq \sum_{j \geq 1} C 2^{-j}=C .
$$

This proves (4.29).

On the one hand, we observe that, $\mathcal{R}(h) \in \mathcal{X}^{\nu+\eta} \subset \mathcal{X}^{\nu}$, therefore, by (4.29) and $i$ ) of Lemma 4.3 we obtain

$$
\|\mathcal{R}(h)\|_{\nu, b} \leq \rho_{1}^{-\eta}\left(1+\gamma^{2}\right)^{\eta / 2}\|\mathcal{R}(h)\|_{\nu+\eta, b} \leq C \rho_{1}^{-\eta}\left(1+\gamma^{2}\right)^{\eta / 2} .
$$

On the other hand, since $\mathcal{R}(h) \in \mathcal{X}^{\nu}$, we deduce that $\partial_{z} \mathcal{B}(\mathcal{R}(h)) \in \mathcal{X}^{\nu}$ using $\left.i i\right)$ of Lemma 4.6, and hence $\mathcal{F}(h) \in \mathcal{X}^{\nu}$. Again by $\left.i i\right)$ of Lemma 4.6, we can bound the norm of $\partial_{z} \mathcal{B}(\mathcal{R}(h))$ and finally using the definitions of $R_{0}$ and $\rho_{1}$ and bound (4.30) of $\|\mathcal{R}(h)\|_{\nu, b}$, we obtain that

$\|\mathcal{F}(h)\|_{\nu, b} \leq\left\|h_{0}\right\|_{\nu, b}+\left\|\partial_{z} \mathcal{B}(\mathcal{R}(h))\right\|_{\nu, b} \leq \frac{R_{0}}{8}+C_{\nu, \gamma} C \rho_{1}^{-\eta}\left(1+\gamma^{2}\right)^{\eta / 2} \leq \frac{R_{0}}{8}+\frac{R_{0}}{16}<\frac{R_{0}}{4}$

and the lemma is proved.

Lemma 4.10 For any $\gamma>0,0<b<b_{0}$, there exists $\tilde{\rho}_{0}=\tilde{\rho}_{0}(\gamma, b, \ell, r)$ such that the fixed point equation

$$
\varphi=\mathcal{F}^{\ell}(\varphi):=\mathcal{B}\left(\psi_{1}^{\ell}\right)+\partial_{z} \mathcal{B}\left(\psi_{2}^{\ell}(\varphi)\right)
$$

has a solution $\varphi \in \mathcal{X}_{\gamma, \tilde{\rho}_{0}, b}^{\ell+1}$. Moreover $\psi_{2}^{\ell}(\varphi) \in \mathcal{X}_{\gamma, \tilde{\rho}_{0}, b}^{\ell+1+\eta_{\ell}}$ with $\eta_{\ell}=\ell-2 r$ if $\ell>2 r$ and $\eta_{\ell}=1$ if $\ell=2 r$.

Proof. The notation used along this proof was introduced in Subsection 4.2.

We fix $\gamma>0,0<b<b_{0}$ and $\rho=\left(1+\gamma^{2}\right)^{1 / 2}+8 \mu_{0} C_{\gamma / 2}^{-1}\left(1+\gamma^{2}\right)^{1 / 2}\left\|F_{1}\right\|_{0, b}>0$. Such choice will be justified later. We notice that since $F_{1}$ does not depend on $z,\left\|F_{1}\right\|_{0, b}$ is independent on $\rho$. 
In order to prove this result, we are going to check that the hypotheses of Lemma 4.9 are satisfied for $\nu=\ell+1, \eta_{\ell}>0, h_{0}=\mathcal{B}\left(\psi_{1}^{\ell}\right)$ and $\mathcal{R}=\psi_{2}^{\ell}$. If the hypotheses of Lemma 4.9 are fulfilled, checking that $\psi_{2}^{\ell}(\varphi) \in \mathcal{X}_{\gamma, \tilde{\rho}_{0}, b}^{\ell+1+\eta_{\ell}}$ is straightforward from (4.29).

We notice that since the functions $Q_{j}$ are $2 \pi$-periodic and analytic in $S_{b_{0}}$, they belong to $\mathcal{X}_{\gamma, s, b}^{0}$ for all $s>0$.

First we deal with the case $\ell>2 r$ which is simpler. By definition (4.18) of $\psi_{1}^{\ell}$, it is clear that $\psi_{1}^{\ell} \in \mathcal{X}^{\ell+1}$. Moreover, $\left\langle\psi_{1}^{\ell}\right\rangle=0$. Therefore, by iii) of Lemma 4.6, $\mathcal{B}\left(\psi_{1}^{\ell}\right) \in \mathcal{X}^{\ell+1}$. As in Lemma 4.9, we define $R_{0}=\left\|\mathcal{B}\left(\psi_{1}^{\ell}\right)\right\|_{\ell+1, b}+1 / 2$.

On the other hand, definition (4.19) of $\psi_{2}^{\ell}$, implies that it is analytic (in fact it is a polynomial in $\varphi), \psi_{2}^{\ell}(0)=0$ and moreover,

$$
\begin{aligned}
& \partial_{\varphi} \psi_{2}^{\ell}(0)=-\mu Q_{1}(\tau) z^{-\ell+2 r} \in \mathcal{X}^{\ell-2 r}, \\
& \partial_{\varphi}^{2} \psi_{2}^{\ell}(0)=-\mu z^{2 r}-2 \mu^{2} z^{4 r-\ell} Q_{2}(\tau) \in \mathcal{X}^{-2 r} \subset \mathcal{X}^{\ell-2 r-(\ell+1)} \\
& \partial_{\varphi}^{j} \psi_{2}^{\ell}(0)=-j ! \mu^{j} z^{2 r j-\ell} Q_{j}(\tau) \in \mathcal{X}^{\ell-2 r j} \subset \mathcal{X}^{\ell-2 r-(j-1)(\ell+1)}, \quad \text { if } 3 \leq j \leq N, \\
& \partial_{\varphi}^{j} \psi_{2}^{\ell}(0)=0, \quad \text { if } j>N,
\end{aligned}
$$

provided $\ell>2 r$. We also notice that, since $\rho \geq\left(1+\gamma^{2}\right)^{1 / 2}$, by $\left.i\right)$ of Lemma 4.3,

$$
\left\|\partial_{\varphi}^{j} \psi_{2}^{\ell}(0)\right\|_{\ell-2 r-(j-1)(\ell+1)} \leq \mu_{0}+j ! \mu_{0}^{j}\left\|Q_{j}\right\|_{0, b}, \quad \text { if } 1 \leq j \leq N .
$$

Hence, the hypotheses of Lemma 4.9 are satisfied by $\mathcal{R}=\psi_{2}^{\ell}$ with $\eta_{\ell}=\ell-2 r, \nu=\ell+1$ and $C=\max _{0 \leq j \leq N}\left(\mu_{0}+\mu_{0}^{j}\left\|Q_{j}\right\|_{0, b}\right)\left(2 R_{0}\right)^{j}$.

Now we deal with the case $\ell=2 r$. First we check that $\mathcal{B}\left(\psi_{1}^{2 r}\right) \in \mathcal{X}^{2 r+1}$. Looking at definition (4.18) of $\psi_{1}^{2 r}$ one deduces that $\psi_{1}^{2 r}=-\chi_{1}+\mathcal{T}_{-\chi_{1}}\left(F_{1}\right)$ where $\chi_{1}$ was defined in (4.15) and $\mathcal{T}_{-\chi_{1}}$ is the operator defined in Lemma 4.4. Hence, since $\chi_{1} \in \mathcal{X}_{\gamma / 2, \rho / 4, b}^{2 r+1}$, again using Lemma 4.4 , we have that $\mathcal{T}_{-\chi_{1}}\left(F_{1}\right) \in \mathcal{X}^{2 r+2}$ provided that $\rho \geq 8 \mu_{0} C_{\gamma / 2}^{-1}\left(1+(\gamma / 2)^{2}\right)^{1 / 2}\left\|F_{1}\right\|_{0, b}$. Therefore, by $\left.i\right)$ of Lemma $4.6, \mathcal{B}\left(\mathcal{T}_{-\chi_{1}}\left(F_{1}\right)\right) \in \mathcal{X}^{2 r+1}$. Finally, we observe that $\left\langle\chi_{1}\right\rangle=0$. Thus iii) of Lemma 4.6 implies that $\mathcal{B}\left(-\chi_{1}\right) \in \mathcal{X}^{2 r+1}$ and henceforth, $\mathcal{B}\left(\psi_{1}^{2 r}\right) \in \mathcal{X}^{2 r+1}$.

Now we check that $\mathcal{R}=\psi_{2}^{2 r}$ satisfies the hypotheses of Lemma 4.9 with $\eta_{\ell}=1$ and $\nu=2 r+1$. Indeed, we note that $\psi_{2}^{2 r}(0)=0$ and, since $z+\mu F_{1}(\tau) \in \mathcal{X}^{1}$,

$\partial_{\varphi}^{1} \psi_{2}^{2 r}(0)=0$

$\partial_{\varphi}^{2} \psi_{2}^{2 r}(0)=-\mu\left(z+\mu F_{1}(\tau)\right)^{2 r}-2 \mu^{2}\left(z+\mu F_{1}(\tau)\right)^{2 r} Q_{2}(\tau) \in \mathcal{X}^{1-(2 r+1)}$

$\partial_{\varphi}^{j} \psi_{2}^{2 r}(0)=-j ! \mu^{j}\left(z+\mu F_{1}(\tau)\right)^{2 r(j-1)} Q_{j}(\tau) \in \mathcal{X}^{1-(j-1)(2 r+1)}$ if $3 \leq j \leq N$,

$\partial_{\varphi}^{j} \psi_{2}^{2 r}(0)=0 \quad$ if $j>N$.

Therefore, using the definition of $\rho$, we realize that

$\left\|\partial_{\varphi}^{j} \psi_{2}^{2 r}(0)\right\|_{1-(j-1)(2 r+1)} \leq\left(\mu_{0}+j ! \mu_{0}^{j}\left\|Q_{j}\right\|_{0, b}\right)\left(1+\frac{C_{\gamma / 2}}{8}\right)^{2 r(j-1)}, \quad$ if $1 \leq j \leq N$

and the proof is complete.

4.4.2. Proof of Proposition 4.8 We fix $\gamma>0,0<b<b_{0}$ and we define $\rho_{0}=$ $\max \left\{4\left(4+\gamma^{2}\right)^{1 / 2} C_{\gamma / 2}^{-1}\left\|F_{1}\right\|_{0, b}, 8 \tilde{\rho}_{0}(\gamma / 4, b, \ell, r)\right\}$, where $\tilde{\rho}_{0}$ was given in Lemma 4.10. This choice of $\rho_{0}$ is justified by the following computations. 
By Lemma 4.10 the fixed point equation (4.31) has a solution $\varphi \in \mathcal{X}_{\gamma / 4, \rho_{0} / 8, b}^{\ell+1}$ such that $\varphi-\mathcal{B}\left(\psi_{1}^{\ell}\right) \in \mathcal{X}_{\gamma / 4, \rho_{0} / 8, b}^{\ell+1+\eta_{\ell}}$. We claim that

$$
\partial_{z} \mathcal{B}\left(\psi_{2}^{\ell}(\varphi)\right)=\mathcal{B}\left(\partial_{z} \psi_{2}^{\ell}(\varphi)\right) \quad \text { on } \quad D_{\gamma / 2, \rho_{0} / 4} \times S_{b}
$$

Indeed, since $\psi_{2}^{\ell}(\varphi) \in \mathcal{X}_{\gamma / 4, \rho_{0} / 8, b}^{\ell+1+\eta_{\ell}}$, using $\left.i i i\right)$ of Lemma 4.3 we get that $\partial_{z} \psi_{2}^{\ell}(\varphi) \in$ $\mathcal{X}_{\gamma / 2, \rho_{0} / 4, b}^{\ell+2+\eta_{\ell}}$. Therefore, by Remark 4.2,

$\left|\partial_{z} \psi_{2}^{\ell}(\varphi)(z, \tau)\right| \leq|z|^{-\ell-2-\eta_{\ell}}\left\|\partial_{z} \psi_{2}^{\ell}(\varphi)\right\|_{\mathcal{X}_{\gamma / 2, \rho_{0} / 4}^{\ell+2+\eta_{\ell}}}$, for all $(z, \tau) \in D_{\gamma / 2, \rho_{0} / 4} \times S_{b}$,

and we obtain (4.32) by differentiating under the integral sign $\mathcal{B}\left(\psi_{2}^{\ell}(\varphi)\right)$.

Equality (4.32) implies that $\varphi$ is a solution of equation $\varphi=\mathcal{B}\left(\psi_{1}^{\ell}\right)+\mathcal{B}\left(\partial_{z} \psi_{2}^{\ell}(\varphi)\right)$ and hence, since $\left(\partial_{\tau}+\partial_{z}\right) \circ \mathcal{B}=\mathrm{Id}$ (Remark 4.5), $\varphi$ is a solution of equation (4.20) belonging to $\mathcal{X}_{\gamma / 2, \rho / 4, b}^{\ell+1}$. Moreover, $\varphi-\mathcal{B}\left(\psi_{1}^{\ell}\right) \in \mathcal{X}_{\gamma / 2, \rho_{0} / 4, b}^{\ell+1+\eta_{\ell}}$.

Taking into account the relation between equations (4.20) and (4.16) given in Subsection 4.2, clearly, if $\ell>2 r$ we deduce that $\varphi$ is a solution of equation (4.16) belonging to $\mathcal{X}_{\gamma / 2, \rho_{0} / 4, b}^{\ell+1} \subset \mathcal{X}_{\gamma, \rho_{0}, b}^{\ell+1}$ and in this case we are done.

In the case $\ell=2 r$, the function $\widetilde{\varphi}(z, \tau)=\varphi\left(z-\mu F_{1}(\tau), \tau\right)$ is a solution of equation (4.16). Moreover, since $\varphi \in \mathcal{X}_{\gamma / 2, \rho_{0} / 4, b}^{2 r+1}$ applying Lemma 4.4 we obtain that $\widetilde{\varphi} \in \mathcal{X}_{\gamma, \rho_{0}, b}^{2 r+1}$ provided that $\rho_{0} \geq 4\left(4+\gamma^{2}\right)^{1 / 2} C_{\gamma / 2}^{-1}\left\|F_{1}\right\|_{0, b}$. We also note that, since $\tilde{\varphi}-\varphi=\mathcal{T}_{\varphi}\left(F_{1}\right) \in \mathcal{X}_{\gamma, \rho_{0}, b}^{2 r+2}$, we have that $\varphi-\mathcal{B}\left(\psi_{1}^{2 r}\right) \in \mathcal{X}_{\gamma, \rho_{0}, b}^{2 r+2}$.

Finally, the uniqueness of the solution $\varphi$ follows from Remark 4.7.

\subsection{End of the proof of Theorem 3.1}

Given $\gamma>0$ and $0<b<b_{0}$, let $\varphi$ be the solution of equation (4.16) belonging to $\mathcal{X}_{\gamma, \rho_{0}, b}^{\ell+1}$, where $\rho_{0}=\rho_{0}(\gamma, b, \ell, r)$ is the constant given by Proposition 4.8.

We claim that the solutions of equation (4.14) such that their derivative with respect to $z$ belong to $\mathcal{X}_{\gamma, \rho_{0}, b}^{\ell+1}$ are defined up to constant. Indeed, let $\phi^{1}, \phi^{2}$ be two solutions of (4.14). Clearly $\partial_{z} \phi^{1}, \partial_{z} \phi^{2}$ are solutions of equation (4.16). Assuming that they belong to $\mathcal{X}_{\gamma, \rho_{0}, b}^{\ell+1}$, by Proposition 4.8, $\partial_{z} \phi^{1}=\partial_{z} \phi^{2}$. Hence $\partial_{\tau}\left(\phi^{1}-\phi^{2}\right)=0$ which implies that $\phi^{1}(z, \tau)=\phi^{2}(z, \tau)+\tilde{\phi}(z)$ and thus, using that $\partial_{z} \phi^{1}=\partial_{z} \phi^{2}$, we conclude that $\partial_{z} \tilde{\phi}=0$.

For any $\xi \in \mathbb{C}$, we define

$$
\phi_{1}(z, \tau)=\int_{+\infty}^{0} \varphi(z+t, \tau) \mathrm{d} t+\xi .
$$

Obviously $\partial_{z} \phi_{1}=\varphi$ and moreover the condition $\varphi \in \mathcal{X}_{\gamma, \rho_{0}, b}^{\ell+1}$ implies $\phi_{1}-\xi \in \mathcal{X}_{\gamma, \rho_{0}, b}^{\ell}$. We note that, by Remark 4.2, we have that $\sup _{(z, \tau) \in D_{\gamma, \rho_{0}} \times S_{b}}\left|z^{\ell+1} \partial_{z} \phi_{1}(z, \tau)\right|<+\infty$.

From the fact that $\varphi$ are solutions of equation (4.16) and $\varphi \in \mathcal{X}_{\gamma, \rho_{0}, b}^{\ell+1}$, it is straightforward to check that $\phi_{1}$ defined by (4.33) are solutions of (4.14) for any $\xi$. This ends the proof of Theorem 3.1. 


\section{Distance between $\partial_{z} \phi^{+}$and $\partial_{z} \phi^{-}$}

Let $\gamma>0$ and $0<b<b_{0}$. We fix $\phi^{ \pm}=\phi_{0}+\mu \phi_{1}^{ \pm}$satisfying the conclusions of Theorem 3.1.

In order to prove Theorem 3.3 we define $\Delta \phi_{1}=\phi_{1}^{-}-\phi_{1}^{+}$. This function has the following immediate properties which come from the ones of $\phi_{1}^{ \pm}$:

i) $\Delta \phi_{1}$ is $2 \pi$-periodic with respect to $\tau$.

ii) It is analytic on $E_{\gamma, \rho_{0}} \times S_{b} \times B\left(\mu_{0}\right)$. This is due to the fact that $E_{\gamma, \rho_{0}} \subset D_{\gamma, \rho_{0}}^{-} \cap D_{\gamma, \rho_{0}}^{+}$. We recall that $E_{\gamma, \rho}$ was defined in (3.1).

iii) $\sup _{(z, \tau) \in E_{\gamma, \rho_{0}} \times S_{b}}\left|z^{\ell+1} \partial_{z} \Delta \phi_{1}\right|<+\infty$.

Since $\phi_{1}^{ \pm}$are necessarily solutions of equation (4.14), subtracting equation (4.14) for $\phi_{1}^{-}$and $\phi_{1}^{+}$respectively we obtain that $\Delta \phi_{1}$ satisfies a partial differential equation of the form

$$
\partial_{\tau} \varphi+\left(1+\mu G^{\ell}(z, \tau)\right) \partial_{z} \varphi=0
$$

where $G^{\ell}$ is an analytic function on $E_{\gamma, \rho_{0}} \times S_{b}, 2 \pi$-periodic on $\tau$, depending not on $\phi_{1}^{ \pm}$ but on $\partial_{z} \phi_{1}^{ \pm}$. Later on, in Subsection 5.2, we will write it in a more detailed way.

Next let us assume that equation (5.1) has a solution $\varphi_{0}$ such that $\psi_{0}(z, \tau)=$ $\left(\varphi_{0}(z, \tau), \tau\right)$ is injective in $E_{\gamma, \rho} \times S_{b}$ for $\rho \geq \rho_{0}$ big enough. We claim that any solution of equation (5.1) defined in $E_{\gamma, \rho} \times S_{b}$ can be written as $\varphi=\chi\left(\varphi_{0}\right)$ for some function $\chi$ (this is a well known property of homogeneous linear partial differential equations). Indeed, we note that, since $\psi_{0}$ is invertible, $\partial_{z} \varphi_{0} \circ \psi_{0}^{-1} \neq 0$ in $E_{\gamma, \rho}$; hence using that both $\varphi$ and $\varphi_{0}$ are solutions of (5.1),

$\partial_{\tau}\left(\varphi \circ \psi_{0}^{-1}\right)=\frac{1}{\partial_{z} \varphi_{0} \circ \psi_{0}^{-1}}\left(-\partial_{z} \varphi \circ \psi_{0}^{-1} \cdot \partial_{\tau} \varphi_{0} \circ \psi_{0}^{-1}+\partial_{\tau} \varphi \circ \psi_{0}^{-1} \cdot \partial_{z} \varphi_{0} \circ \psi_{0}^{-1}\right)=0$.

Therefore, $\varphi \circ \psi_{0}^{-1}(\xi, \tau)$ does not depend on $\tau$ and this implies that there exists a function $\chi$ such that $\varphi \circ \psi_{0}^{-1}(\xi, \tau)=\chi(\xi)$ and the claim is proved evaluating this equality at $\xi=\varphi_{0}(z, \tau)$.

Subsection 5.4 is devoted to proving the existence and useful properties of such a solution $\varphi_{0}$ of equation (5.1). Specifically we will prove that there exists a solution of the form $\varphi_{0}(z, \tau)=z-\tau+\mu g(z, \tau)$, with $g 2 \pi$-periodic with respect to $\tau$ and satisfying all the properties stated in Theorem 3.3. We will also prove that $\lim _{\operatorname{Im} z \rightarrow-\infty} \partial_{z} g(z, \tau)=0$. Finally we will see that $\left(\varphi_{0}(z, \tau), \tau\right)$ is injective in $E_{\gamma, \rho_{1}}$ with $\rho_{1} \geq \rho_{0}$ big enough.

In Subsection 5.5, using this fact and properties i), ii) and iii) that $\Delta \phi_{1}$ satisfies, we will end the proof of Theorem 3.3. We sketch the process we will follow below.

On the one hand, since $\Delta \phi_{1}$ is a solution of equation (5.1) analytic in $E_{\gamma, \rho_{1}} \times$ $S_{b} \times B\left(\mu_{0}\right)$ (property ii)), there exists an analytic function $\chi$ such that $\Delta \phi_{1}(z, \tau)=$ $\chi(z-\tau+\mu g(z, \tau))$. On the other hand, $\Delta \phi_{1}$ is $2 \pi$-periodic with respect to $\tau$. This implies that $\chi$ have to be a $2 \pi$-periodic function in such a way that $\Delta \phi_{1}$ has to be of the form

$$
\Delta \phi_{1}(z, \tau)=\sum_{k \in \mathbb{Z}} \chi_{k}(\mu) \mathrm{e}^{\mathrm{i} k(z-\tau+\mu g(z, \tau))}
$$


(We notice that, $\chi_{k}$ are analytic functions in $B\left(\mu_{0}\right)$.) Finally, using that $\Delta \phi_{1}$ goes to 0 as $\operatorname{Im} z \rightarrow-\infty$ (property iii)), one can check that $\chi_{k}=0$ for $k>0$. Henceforth

$$
\partial_{z} \Delta \phi_{1}(z, \tau)=\sum_{k<0} \mathrm{i} k \chi_{k}(\mu) \mathrm{e}^{\mathrm{i} k(z-\tau+\mu g(z, \tau))}\left(1+\mu \partial_{z} g(z, \tau)\right)
$$

and since $\lim _{\operatorname{Im} z \rightarrow-\infty} \partial_{z} g(z, \tau)=0$, we obtain the asymptotic expression (3.5) of Theorem 3.3 as a consequence of (5.2).

In order to obtain (3.6) we need only to look for the independents of $\mu$ terms of the functions $\partial_{z} \phi_{1}^{ \pm}$and compute their difference.

The Subsection below is devoted to introduce the notation we will use throughout this Section.

\subsection{Notation}

As in Section 4 , we will denote by $\langle\cdot\rangle$ the mean with respect to $\tau$. For any $2 \pi$-periodic function, $h$, we also introduce $\{h\}=h-\langle h\rangle$.

Now we introduce the Banach spaces we deal with during this Section. These spaces will be analogous to the ones defined in Subsection 4.1 for functions defined on the domain $E_{\gamma, \rho} \times S_{b} \times B\left(\mu_{0}\right)$ (we recall that $E_{\gamma, \rho}$ was defined in (3.1)). We observe that the function $\Delta \phi_{1}=\phi_{1}^{-}-\phi_{1}^{+}$is defined on such complex domain.

For any $\gamma, \rho, b>0$ and $\nu \in \mathbb{R}$, to shorten the notation, we will write

$$
\bar{E}_{\gamma, \rho}=E_{\gamma, \rho} \times B\left(\mu_{0}\right), \quad \mathcal{E}_{\gamma, \rho, b}=E_{\gamma, \rho} \times S_{b} \times B\left(\mu_{0}\right) .
$$

We define the spaces

$Y^{\nu}=\left\{h: \bar{E}_{\gamma, \rho} \rightarrow \mathbb{C}: h\right.$ is analytic and $\left.\|h\|_{\nu}:=\sup _{(z, \mu) \in \bar{E}_{\gamma, \rho}}\left|z^{\nu} h(z, \mu)\right|<+\infty\right\}$

if $\nu \neq 0$ and

$Y^{0}=\left\{h: \bar{E}_{\gamma, \rho} \rightarrow \mathbb{C}: h\right.$ is analytic and $\left.\|h\|_{0}:=\sup _{(z, \mu) \in \bar{E}_{\gamma, \rho}} \frac{|h(z, \mu)|}{|\log | z||}<+\infty\right\}$

for $\nu=0$. It is clear that the functional spaces $Y^{\nu}$ equipped with the norm $\|\cdot\|_{\nu}$ are Banach spaces.

We also introduce the spaces of Fourier series

$$
\begin{gathered}
\mathcal{Y}_{\gamma, \rho, b}^{\nu}=\left\{f: \mathcal{E}_{\gamma, \rho, b} \rightarrow \mathbb{C}: \text { analytic, } f(z, \tau, \mu)=\sum_{k \in \mathbb{Z}} f_{k}(z, \mu) \mathrm{e}^{\mathrm{i} k \tau}, f_{k} \in Y^{\nu}\right. \\
\text { and } \left.\|f\|_{\nu, b}:=\sum_{k \in \mathbb{Z}}\left\|f_{k}\right\|_{\nu} \mathrm{e}^{|k| b}<+\infty\right\}
\end{gathered}
$$

The functional space $\mathcal{Y}_{\gamma, \rho, b}^{\nu}$ of Fourier series endowed with the norm $\|\cdot\|_{\nu, b}$ is a Banach space.

We also define the auxiliary Banach space

$$
\begin{gathered}
\overline{\mathcal{Y}}_{\gamma, \rho, b}^{0}=\left\{f: \mathcal{E}_{\gamma, \rho, b} \rightarrow \mathbb{C}: \text { analytic, } f(z, \tau, \mu)=\sum_{k \in \mathbb{Z}} f_{k}(z, \mu) \mathrm{e}^{\mathrm{i} k \tau}\right. \\
\text { and } \left.\|f\|_{\overline{0}, b}:=\sum_{k \in \mathbb{Z}} \sup _{(z, \mu) \in \bar{E}_{\gamma, \rho}}\left|f_{k}(z, \mu)\right| \mathrm{e}^{|k| b}<+\infty\right\} .
\end{gathered}
$$


For notational need we introduce $\overline{\mathcal{Y}}_{\gamma, \rho, b}^{\nu}=\mathcal{Y}_{\gamma, \rho, b}^{\nu}$ and $\|\cdot\|_{\bar{\nu}, b}=\|\cdot\|_{\nu, b}$ if $\nu \neq 0$.

Remark 5.1 We notice that $\mathcal{X}^{\nu, \pm} \subset \mathcal{Y}^{\nu}$ and $\mathcal{X}^{0, \pm} \subset \overline{\mathcal{Y}}^{0}$.

Let $\phi^{ \pm}=\phi_{0}+\phi_{1}^{ \pm}$satisfying the conclusions of Theorem 3.1. We notice that, by Remark 4.2, $\partial_{z} \phi_{1}^{ \pm} \in \mathcal{X}_{\gamma, \rho, b}^{\ell+1, \pm}$ respectively, for any $\gamma>0, \rho \geq \rho_{0}$ and $0<b<b_{0}$. Hence the function $\Delta \phi_{1}=\phi_{1}^{-}-\phi_{1}^{+}$satisfies that $\partial_{z} \Delta \phi_{1}$ belongs to $\mathcal{Y}_{\gamma, \rho, b}^{\ell+1}$.

We will denote $\mathcal{Y}_{\gamma, \rho, b}^{\nu}$ simply by $\mathcal{Y}^{\nu}$ (and $\overline{\mathcal{Y}}_{\gamma, \rho, b}^{\nu}$ by $\overline{\mathcal{Y}}^{\nu}$ ) is there is no danger of confusion on the definition domain. We will also write $\|\cdot\|_{\mathcal{Y}_{\gamma, \rho, b}^{\nu}}=\|\cdot\|_{\nu, b}$ and $\|\cdot\|_{\overline{\mathcal{Y}}_{\gamma, \rho, b}^{\nu}}=\|\cdot\|_{\bar{\nu}, b}$, when it is necessary to emphasize the complex domain where the functions are defined.

The Banach spaces $\overline{\mathcal{Y}}^{\nu}$ (and henceforth, $\mathcal{Y}^{\nu}$ for $\nu \neq 0$ ), satisfy the same properties as the ones given in Subsection 4.1 for $\mathcal{X}^{\nu}$. Specifically we have the following lemma.

Lemma 5.2 Let $\gamma, b, \rho>0$ and $\nu, \eta \in \mathbb{R}$.

i) If $\eta \geq \nu$, then $\mathcal{Y}^{\eta} \subset \mathcal{Y}^{\nu}$ and $\overline{\mathcal{Y}}^{\eta} \subset \overline{\mathcal{Y}}^{\nu}$. Moreover we have that

$$
\|h\|_{\bar{\nu}, b} \leq \rho^{\nu-\eta}\|h\|_{\bar{\eta}, b}, \quad \text { if } h \in \overline{\mathcal{Y}}^{\nu} .
$$

ii) If $h \in \overline{\mathcal{Y}}^{\nu}$ and $g \in \overline{\mathcal{Y}}^{\eta}$, then the product $h g \in \overline{\mathcal{Y}}^{\nu+\eta}$ and

$$
\|h g\|_{\overline{\nu+\eta}, b} \leq\|h\|_{\bar{\nu}, b}\|g\|_{\bar{\eta}, b} .
$$

iii) If $h \in \overline{\mathcal{Y}}_{\gamma, \rho, b}^{\nu}$, then there exists a constant $A_{\gamma, \nu}$ such that for $l \in \mathbb{N} \backslash\{0\}$ we have that

$$
\partial_{z}^{l} h \in \overline{\mathcal{Y}}_{2 \gamma, 2 \rho, b}^{l+\nu} \quad \text { and } \quad\left\|\partial_{z}^{l} h\right\|_{\overline{l+\nu}, b} \leq l ! 2^{-l} C_{\gamma}^{-l} A_{\gamma, \nu}\|h\|_{\overline{\mathcal{Y}}_{\gamma, \rho, b}^{\nu}}
$$

where the constant $C_{\gamma}$ was defined by (4.8).

iv) Assume that $\eta>-1$. We fix $F \in \overline{\mathcal{Y}}_{\gamma, \rho, b}^{\nu}$ and $f \in \overline{\mathcal{Y}}_{\gamma, \rho, b}^{\eta}$. Let $\rho$ be such that

$$
\mu_{0} C_{\gamma}^{-1}\|f\|_{\overline{\mathcal{Y}}_{\gamma, \rho, b}^{\eta}} \leq \rho^{\eta+1} \text {. }
$$

We define the formal expression

$$
\mathcal{T}_{F}(f)(z, \tau)=\sum_{l \geq 1} \frac{1}{l !} \partial_{z}^{l} F(z, \tau) \mu^{l} f^{l}(z, \tau) .
$$

Then there exists a constant $B_{\gamma, \nu}$ such that

$$
\mathcal{T}_{F}(f) \in \overline{\mathcal{Y}}_{2 \gamma, 2 \rho, b}^{\nu+\eta+1}, \quad\left\|\mathcal{T}_{F}(f)\right\|_{\overline{\nu+\eta+1}, b} \leq \mu_{0} B_{\gamma, \nu}\|F\|_{\overline{\mathcal{Y}}_{\gamma, \rho, b}^{\nu}}\|f\|_{\overline{\mathcal{Y}}_{\gamma, \rho, b}^{\eta}} .
$$

Moreover, the function $\tilde{F}(z, \tau)=F(z+\mu f(z, \tau), \tau)$ belongs to $\overline{\mathcal{Y}}_{2 \gamma, 2 \rho, b}^{\nu}$.

v) Let $\eta>-1, F \in \mathcal{Y}_{\gamma, \rho, b}^{0}$ and $f \in \overline{\mathcal{Y}}_{\gamma, \rho, b}^{\eta}$ with $F$ satisfying that $\partial_{z} F \in \mathcal{Y}_{\gamma, \rho, b}^{1}$. If $\rho$ satisfies (5.4), then

$$
\mathcal{T}_{F}(f) \in \mathcal{Y}_{2 \gamma, 2 \rho, b}^{\eta+1}, \quad\left\|\mathcal{T}_{F}(f)\right\|_{\eta+1, b} \leq \mu_{0} B_{\gamma, 0}\left\|\partial_{z} F\right\|_{\mathcal{Y}_{\gamma, \rho, b}^{1}}\|f\|_{\overline{\mathcal{Y}}_{\gamma, \rho, b}^{\eta}} .
$$

Moreover $\tilde{F}(z, \tau)=F(z+\mu f(z, \tau), \tau)$ belongs to $\mathcal{Y}_{2 \gamma, 2 \rho, b}^{0}$.

Proof. The proof of $i$ ), ii) and $i i i$ ) of Lemma 5.2 is completely analogous to the proof of Lemma 4.3. We only have to take into account two facts: the first is that if $z \in E_{\gamma, \rho}$, then $|z| \geq \rho$; the second we need is that

$$
\left\{u \in \mathbb{C}:|u-z|<2 C_{\gamma}|z|\right\} \subset E_{\gamma, \rho}, \quad z \in E_{2 \gamma, 2 \rho} .
$$

The $i v$ ) item is proved as in Lemma 4.3 by using (5.5). To check $v$ ) we apply iii) to $\partial_{z} F$ and we proceed in a completely analogous way to the one in $i v$ ) of Lemma 4.3 . 


\subsection{The equation for $\phi_{1}^{-}-\phi_{1}^{+}$}

Let $\gamma>0$ and $0<b<b_{0}$. According to Theorem 3.1 there exist infinitely many solutions $\phi^{ \pm}=\phi_{0}+\mu \phi_{1}^{ \pm}$of the Hamilton-Jacobi equation (3.3) analytic on the domain $D_{\gamma, \rho_{0}}^{ \pm} \times S_{b}, 2 \pi$-periodic with respect to $\tau$ and such that $\partial_{z} \phi_{1}^{ \pm}$are the unique possible choices satisfying that

$$
\sup _{(z, \tau) \in D_{\gamma, \rho_{0}}^{ \pm} \times S_{b}}\left|z^{\ell+1} \partial_{z} \phi_{1}^{ \pm}(z, \tau)\right|<+\infty .
$$

We recall that $\rho_{0}=\rho_{0}(\gamma, b, \ell, r)$ was given in Theorem 3.1.

For any two of those solutions $\phi^{ \pm}$we denote $\Delta \phi_{1}=\phi_{1}^{-}-\phi_{1}^{+}$which is defined on $E_{\gamma, \rho_{0}} \times S_{b} \subset D_{\gamma, \rho_{0}}^{+} \cap D_{\gamma, \rho_{0}}^{-} \times S_{b}$. Since, $\phi_{1}^{ \pm}$are solutions of (4.14), subtracting equation (4.14) for both $\phi_{1}^{-}$and $\phi_{1}^{+}$respectively we get

$$
\partial_{\tau} \Delta \phi_{1}+\partial_{z} \Delta \phi_{1}+\mathcal{H}_{1}\left(z, \partial_{z} \phi^{-}, \tau\right)-\mathcal{H}_{1}\left(z, \partial_{z} \phi^{+}, \tau\right)+\frac{\mu}{2} z^{2 r}\left[\left(\partial_{z} \phi_{1}^{-}\right)^{2}-\left(\partial_{z} \phi_{1}^{+}\right)^{2}\right]=0 .
$$

Denoting

$G^{\ell}(z, \tau)=\sum_{j=1}^{N} \mu^{j-1} Q_{j}(\tau) z^{2 r j-\ell} \sum_{k=0}^{j-1}\left(\partial_{z} \phi_{1}^{-}\right)^{k}\left(\partial_{z} \phi_{1}^{+}\right)^{j-1-k}+\frac{1}{2} z^{2 r}\left(\partial_{z} \phi_{1}^{-}+\partial_{z} \phi_{1}^{+}\right)$,

it is straightforward to see that $\Delta \phi_{1}$ satisfies the equation:

$$
\partial_{\tau} \varphi+\left(1+\mu G^{\ell}(z, \tau)\right) \partial_{z} \varphi=0 .
$$

Lemma 5.3 The function $G^{\ell}-Q_{1} z^{-\ell+2 r}$ belongs to $\mathcal{Y}^{\ell-2 r+1}$ and it can be written in the form

$G^{\ell}(z, \tau)=Q_{1}(\tau) z^{-\ell+2 r}+\frac{1}{2} z^{2 r}\left(\partial_{z} \phi_{1}^{-}+\partial_{z} \phi_{1}^{+}\right)\left(1+2 z^{-\ell+2 r} \mu Q_{2}(\tau)\right)+\bar{G}^{\ell}(z, \tau)$

with $Q_{1}$ having zero mean with respect to $\tau$ and $\bar{G}^{\ell} \in \mathcal{Y}^{3(\ell-2 r)+2}$. Moreover

$$
\left\langle G^{\ell}\right\rangle \in \begin{cases}\mathcal{Y}^{2} & \text { if } \ell=2 r, Q_{1}=0, \text { and }\left\langle F_{0} \cdot Q_{2}\right\rangle=0 \\ \mathcal{Y}^{\ell-2 r+1} & \text { otherwise, }\end{cases}
$$

where $F_{0}$ is such that $\partial_{\tau} F_{0}=Q_{0}$ and $\left\langle F_{0}\right\rangle=0$.

Proof. Formula (5.8) is straightforward from definition of $G^{\ell}$. Moreover, using that $\partial_{z} \phi_{1}^{ \pm} \in \mathcal{X}^{\ell+1, \pm}$, we easily get that $\bar{G}^{\ell} \in \mathcal{Y}^{3(\ell-2 r)+2}$.

Now we deal with the statement related to $\left\langle G^{\ell}\right\rangle$. We observe that $G^{\ell}-Q_{1} z^{\ell-2 r} \in$ $\mathcal{Y}^{\ell-2 r+1}$. Hence, since $Q_{1}$ has zero mean, in any case we have that $\left\langle G^{\ell}\right\rangle \in \mathcal{Y}^{\ell-2 r+1}$. It only remains to check that if $\ell=2 r, Q_{1}=0$ and $\left\langle F_{0} \cdot Q_{2}\right\rangle=0,\left\langle G^{\ell}\right\rangle \in \mathcal{Y}^{2}$. First we claim that, in this case,

$$
\partial_{z} \phi_{1}^{ \pm}-2 r \frac{F_{0}}{z^{2 r+1}} \in \mathcal{Y}^{2 r+2} .
$$

Indeed, we deal with the + case, being the - case analogous. By Proposition 4.8, $\partial_{z} \phi_{1}^{+}-\mathcal{B}\left(\psi_{1}^{2 r}\right) \in \mathcal{X}^{2 r+2,+}$. Trivially,

$$
\mathcal{B}\left(\psi_{1}^{2 r}\right)=2 r \int_{+\infty}^{0} \frac{Q_{0}(\tau+t)}{(z+t)^{2 r+1}} \mathrm{~d} t=2 r \frac{F_{0}(\tau)}{z^{2 r+1}}+2 r(2 r+1) \mathcal{B}\left(F_{0} z^{-2 r-2}\right)
$$


and therefore, $\mathcal{B}\left(\psi_{1}^{2 r}\right)-2 r F_{0}(\tau) z^{-2 r-1} \in \mathcal{X}^{2 r+2,+} \subset \mathcal{Y}^{2 r+2}$ because $F_{0}$ has zero mean.

Looking at expression (5.8) of $G^{\ell}$, and using (5.10), we deduce that

$$
G^{\ell}-4 r z^{-1} F_{0}\left(1+\mu Q_{2}\right) \in \mathcal{Y}^{2}
$$

and henceforth, (5.9) is proved provided that $\left\langle F_{0}\right\rangle=\left\langle F_{0} \cdot Q_{2}\right\rangle=0$.

Remark 5.4 Since $G^{\ell}$ depends on $\partial_{z} \phi_{1}^{ \pm}$, but not on $\phi_{1}^{ \pm}$themselves, it is independent of the choice of the solutions $\phi^{ \pm}$.

As we pointed out at the beginning of this Section, the next step to prove Theorem 3.3 is to find a solution of the partial differential equation (5.7) of the form $\varphi_{0}(z, \tau)=z-\tau+\mu g(z, \tau)$. To obtain such a solution, we will need to solve explicitly the linear equation $\partial_{\tau} h+\partial_{z} h=\psi$ with $\psi \in \mathcal{Y}^{\nu}$ a known function. The next Subsection is devoted to studying this equation.

\subsection{An explicit solution of equation $\partial_{\tau} h+\partial_{z} h=\psi$ in $\mathcal{Y}_{\gamma, \rho, b}^{\nu}$}

We fix $b, \rho, \gamma>0, \nu \geq 0$ and $\psi \in \mathcal{Y}^{\nu}$. We denote by $\psi_{k}$ the $k$-Fourier coefficient of $\psi$ and we consider the operator $\mathcal{G}$ formally defined by

$$
\mathcal{G}(\psi)(z, \tau)=\sum_{k \in \mathbb{Z}}(\mathcal{G}(\psi))_{k}(z) \mathrm{e}^{\mathrm{i} k \tau}
$$

where its Fourier coefficients are given by:

$$
\begin{array}{ll}
(\mathcal{G}(\psi))_{0}(z)=\int_{-\mathrm{i} \rho}^{z} \psi_{0}(t) \mathrm{d} t & \text { if } 0 \leq \nu \leq 1 \\
(\mathcal{G}(\psi))_{0}(z)=\int_{-\mathrm{i} \infty}^{z} \psi_{0}(t) \mathrm{d} t & \text { if } \nu>1 \\
(\mathcal{G}(\psi))_{k}(z)=\int_{-\mathrm{i} \rho}^{z} \mathrm{e}^{\mathrm{i} k(t-z)} \psi_{k}(t) \mathrm{d} t & \text { if } k>0 \\
(\mathcal{G}(\psi))_{k}(z)=\int_{-\mathrm{i} \infty}^{z} \mathrm{e}^{\mathrm{i} k(t-z)} \psi_{k}(t) \mathrm{d} t & \text { if } k<0 .
\end{array}
$$

The following lemma proves that, under suitable conditions, $\mathcal{G}(\psi)$ is well defined. This implies that $h=\mathcal{G}(\psi)$ is a solution of equation $\partial_{\tau} h+\partial_{z} h=\psi$.

Lemma 5.5 Let $\gamma, b, \nu>0$ and $\rho \geq \max \{2 \nu, 1\}$. For any $\psi \in \mathcal{Y}_{\gamma, \rho, b}^{\nu}$ we have that

i) $\mathcal{G}(\psi) \in \mathcal{Y}_{\gamma, \rho, b}^{\nu-1}$ and $\partial_{u} \mathcal{G}(\psi) \in \mathcal{Y}_{\gamma, \rho, b}^{\nu}$. Moreover, there exists a constant $C_{\nu, \gamma}$ only depending on $\nu$ and $\gamma$ such that,

$$
\|\mathcal{G}(\psi)\|_{\nu-1, b} \leq C_{\nu, \gamma}\|\psi\|_{\nu, b} \text { and }\left\|\partial_{u} \mathcal{G}(\psi)\right\|_{\nu, b} \leq C_{\nu, \gamma}\|\psi\|_{\nu, b} .
$$

ii) If $\psi$ has zero mean with respect to $\tau$, then $\mathcal{G}(\psi) \in \mathcal{Y}_{\gamma, \rho, b}^{\nu},\langle\mathcal{G}(\psi)\rangle=0$ and

$$
\|\mathcal{G}(\psi)\|_{\nu, b} \leq C_{\nu, \gamma}\|\psi\|_{\nu, b} .
$$

Proof. We write $h=\mathcal{G}(\psi)$ and we denote by $h_{k}$ its $k$-Fourier coefficient. To prove $i$ ) and $i i$ ) we have to bound $h_{k}$. We claim that for all $z \in E_{\gamma, \rho}$, 
(a) If either $k<0$ and $\nu>0$, or $k=0$ and $\nu>1$,

$$
\left|h_{k}(z)\right| \leq\left\|\psi_{k}\right\|_{\nu} \int_{-\infty}^{0} \frac{\mathrm{e}^{-k t}}{\left(|z|^{2}+t^{2}\right)^{\nu / 2}} \mathrm{~d} t .
$$

(b) Otherwise, denoting $c_{\gamma}=\gamma^{-1}\left(1+\gamma^{2}\right)^{1 / 2}$,

$$
\left|h_{k}(z)\right| \leq c_{\gamma}\left\|\psi_{k}\right\|_{\nu} \int_{0}^{|\operatorname{Im} z+\rho|} \frac{\mathrm{e}^{-k t}}{|t+\operatorname{Im} z|^{\nu}} \mathrm{d} t .
$$

Indeed, in case (a) $h_{k}$ is defined by (5.15) and (5.13) respectively. The condition $\psi \in \mathcal{Y}_{\gamma, \rho, b}^{\nu}$ implies that $\mathrm{e}^{\mathrm{i} k s} \psi_{k}(s)$ is analytic on $E_{\gamma, \rho}$ and $\lim _{\operatorname{Im} s \rightarrow-\infty} \mathrm{e}^{\mathrm{i} k s} s \psi_{k}(s)=0$ (either if $k=0$ and $\psi_{0} \in \mathcal{Y}_{\gamma, \rho, b}^{\nu}$ with $\nu>1$, or $k<0$ and $\psi_{k} \in \mathcal{Y}_{\gamma, \rho, b}^{\nu}$ with $\nu>0$ ). Thus, by Cauchy's theorem we can change the path of integration to $z+\mathrm{i} t$ and therefore

$$
\left|h_{k}(z)\right| \leq\left|\mathrm{i} \int_{-\infty}^{0} \mathrm{e}^{-k t} \psi_{k}(z+\mathrm{i} t) \mathrm{d} t\right| \leq\left\|\psi_{k}\right\|_{\nu} \int_{-\infty}^{0} \frac{\mathrm{e}^{-k t}}{|z+\mathrm{i} t|^{\nu}} \mathrm{d} t .
$$

Finally, since $|z+\mathrm{i} t|^{2} \geq|z|^{2}+t^{2}$ if $t<0$, we get (5.17). In case (b), bounding (5.12) and (5.14),

$$
\left|h_{k}(z)\right| \leq \frac{|z+\mathrm{i} \rho|}{|\operatorname{Im} z+\rho|}\left\|\psi_{k}\right\|_{\nu} \int_{0}^{|\operatorname{Im} z+\rho|} \frac{\mathrm{e}^{-k t}}{|t+\operatorname{Im} z|^{\nu}} \mathrm{d} t
$$

and, since $|z+\mathrm{i} \rho||\operatorname{Im} z+\rho|^{-1} \leq c_{\gamma},(5.18)$ holds.

Now we claim that

$$
\left\|h_{k}\right\|_{\nu} \leq 2 c_{\gamma}^{\nu+1}|k|^{-1}\left\|\psi_{k}\right\|_{\nu} \quad \text { for } k \neq 0 \text {. }
$$

Indeed, let $z \in E_{\gamma, \rho}$. First we deal with $k<0$. Obviously, bound (5.17) implies that

$$
\left|z^{\nu} h_{k}(z)\right| \leq\left\|\psi_{k}\right\|_{\nu} \int_{-\infty}^{0} \mathrm{e}^{-k t} \mathrm{~d} t=|k|^{-1}\left\|\psi_{k}\right\|_{\nu} \leq 2 c_{\gamma}^{\nu+1}|k|^{-1}\left\|\psi_{k}\right\|_{\nu}
$$

provided that $c_{\gamma}>1$. If $k>0$ we define $I_{\nu}=\int_{0}^{|\operatorname{Im} z+\rho|} \mathrm{e}^{-k t}|t+\operatorname{Im} z|^{-\nu} \mathrm{d} t$. Integrating by parts $I_{\nu}$, it is easily checked that $I_{\nu} \leq k^{-1}\left(|\operatorname{Im} z|^{-\nu}+\nu \rho^{-1} I_{\nu}\right)$. Thus, since $\rho \geq 2 \nu$, we obtain bound (5.19) from (5.18) by using the fact that $|z| \leq c_{\gamma}|\operatorname{Im} z|$.

We prove $i$ ). Let $\nu>0$. We define the constants $B_{\nu, \gamma}=(1-\nu)^{-1} c_{\gamma}$ if $\nu<1$, $B_{1, \gamma}=c_{\gamma}$ and $B_{\nu, \gamma}=\int_{0}^{+\infty}\left(1+s^{2}\right)^{-\nu / 2} \mathrm{~d} s$ if $\nu>1$. With this notation

$$
\left\|h_{0}\right\|_{\nu-1} \leq B_{\nu, \gamma}\left\|\psi_{0}\right\|_{\nu}
$$

Proving (5.20) is straightforward by computing the integrals in formulae (5.17) and (5.18) in the corresponding cases. We take $C_{\nu, \gamma}=\max \left\{1+2 c_{\gamma}^{\nu+1}, B_{\nu, \gamma}\right\}$ and we notice that bounds (5.20), (5.19) and $i$ ) of Lemma 4.3 imply

$$
\begin{aligned}
\|h\|_{\nu-1, b} & =\left\|h_{0}\right\|_{\nu-1}+\sum_{k \in \mathbb{Z} \backslash\{0\}}\left\|h_{k}\right\|_{\nu-1} \mathrm{e}^{|k| b} \\
& \leq B_{\nu, \gamma}\left\|\psi_{0}\right\|_{\nu}+2 c_{\gamma}^{\nu+1} \rho^{-1} \sum_{k \in \mathbb{Z} \backslash\{0\}}\left\|\psi_{k}\right\|_{\nu} \mathrm{e}^{|k| b} \leq C_{\nu, \gamma}\|\psi\|_{\nu, b}
\end{aligned}
$$

provided that $\rho \geq 1$. In this way we get the first bound of (5.16). 
Next we prove the second bound of (5.16). Taking derivatives in (5.12)-(5.15),

$$
\partial_{z} h_{k}=-\mathrm{i} k h_{k}+\psi_{k}, \quad \text { for all } k \in \mathbb{Z} .
$$

Hence we have that $\partial_{z} h_{0}=\psi_{0}$ and, from (5.19), $\left\|\partial_{z} h_{k}\right\|_{\nu} \leq\left(1+2 c_{\gamma}^{\nu+1}\right)\left\|\psi_{k}\right\|_{\nu}$ for $k \neq 0$. From the Fourier series of $\partial_{z} h$ and the definition of the norm $\|\cdot\|_{\nu, b}$, we get $\left\|\partial_{z} h\right\|_{\nu, b} \leq C_{\nu, \gamma}\|\psi\|_{\nu, b}$.

Finally, we prove $i i$ ). Let $\nu>0$. We observe that, $\langle\psi\rangle=0$ implies $h_{0}=0$. Thus ii) follows from (5.19).

Remark 5.6 By using equality (5.21) one checks that $\mathcal{G}(\psi)$ is a solution of equation $\partial_{\tau} h+\partial_{z} h=\psi$.

\subsection{A solution $\varphi_{0}$ of equation (5.7) of the form $\varphi_{0}(z, \tau)=z-\tau+\mu g(z, \tau)$}

Our goal in this subsection is to prove the following result:

Proposition 5.7 Let $\gamma>0$ and $0<b<b_{0}$. There exists $\rho_{1}=\rho_{1}(\gamma, b, \ell, r)$ such that the equation (5.7):

$$
\partial_{\tau} \varphi+\partial_{z} \varphi\left(1+\mu G^{\ell}\right)=0
$$

has a solution $\varphi_{0}$ of the form $\varphi_{0}(z, \tau)=z-\tau+\mu g(z, \tau)$ with $g$ satisfying that $g \in \mathcal{Y}_{\gamma, \rho_{1}, b}^{\ell-2 r}$ and $\partial_{z} g \in \mathcal{Y}_{\gamma, \rho_{1}, b}^{\ell-2 r+1}$.

In the special case that $\ell=2 r, Q_{1}=0$ and $\left\langle F_{0} \cdot Q_{2}\right\rangle=0$, then $g \in \mathcal{Y}_{\gamma, \rho_{1}, b}^{1}$ and $\partial_{z} g \in \mathcal{Y}_{\gamma, \rho_{1}, b}^{2}$.

Moreover, $\psi_{0}(z, \tau)=\left(\varphi_{0}(z, \tau), \tau\right)$ defines an injective map on $E_{\gamma, \rho_{1}}$.

A function $\varphi_{0}$ is a solution of equation (5.22) of the form $\varphi_{0}(z, \tau)=z-\tau+\mu g(z, \tau)$ if and only if $g$ is a solution of the equation

$$
\partial_{\tau} g+\partial_{z} g\left(1+\mu G^{\ell}\right)=-G^{\ell} .
$$

To check that equation (5.23) has solutions satisfying the conclusions of Proposition 5.7 we state a technical lemma which will be proved later.

Lemma 5.8 We fix $\gamma, b, \rho>0$. Let $H \in \mathcal{Y}_{\gamma / 2, \rho, b}^{\eta}$ be such that $\mathcal{G}(H) \in \mathcal{Y}_{\gamma / 2, \rho, b}^{\nu}$ for some $\eta>0$ and $\nu \geq 0$.

If either $\nu>0$, or $\nu=0$ and $\eta \geq 1$, there exists $\rho_{2}=\rho_{2}(\gamma, b, \nu, \eta, \rho)$ such that the equation

$$
\partial_{\tau} h+\partial_{z} h(1+\mu H)=-H
$$

has a solution $h \in \mathcal{Y}_{\gamma, \rho_{2}, b}^{\nu}$ satisfying that $\partial_{z} h \in \mathcal{Y}_{\gamma, \rho_{2}, b}^{\nu+1}$.

To prove Proposition 5.7 from Lemma 5.8 we have to check that in each case $G^{\ell}$ satisfies the hypotheses of this Lemma.

Proof of Proposition 5.7. We fix $\gamma>0$ and $0<b<b_{0}$. We are forced to distinguish three cases: 
- Case $\ell>2 r$. By Lemma 5.3, $G^{\ell}=Q_{1} z^{-\ell-2 r}+\tilde{G}^{\ell}$ with $\tilde{G}^{\ell} \in \mathcal{Y}_{\gamma, \rho_{0}, b}^{\ell-2 r+1}$. Therefore $G^{\ell} \in \mathcal{Y}_{\gamma, \rho_{0}, b}^{\ell-2 r}$. Moreover, since $Q_{1}$ has zero mean with respect to $\tau$, by Lemma 5.5, $\mathcal{G}\left(G^{\ell}\right) \in \mathcal{Y}_{\gamma, \rho_{0}, b}^{\ell-2 r}$ and Lemma 5.8 can be applied in this case with $H=G^{\ell}$ and $\nu=\eta=\ell-2 r>0$.

- Case $\ell=2 r, Q_{1}=0$ and $\left\langle F_{0} \cdot Q_{2}\right\rangle=0$. Again using Lemma 5.3 one deduce that $G^{\ell}=\left\langle G^{\ell}\right\rangle+\left\{G^{\ell}\right\}$ with $\left\langle G^{\ell}\right\rangle \in \mathcal{Y}_{\gamma, \rho_{0}, b}^{2}$ and $\left\{G^{\ell}\right\} \in \mathcal{Y}_{\gamma, \rho_{0}, b}^{1}$ having zero mean with respect to $\tau$. Using similar arguments as in the previous case, we conclude that we can apply Lemma 5.8 with $H=G^{\ell}$ and $\nu=\eta=1$.

- Case $\ell=2 r$ but either $Q_{1} \neq 0$ or $\left\langle F_{0} \cdot Q_{2}\right\rangle \neq 0$. The change of coordinates

$$
z=u+\mu F_{1}(\tau), \quad \tilde{g}(u, \tau)=g\left(u+\mu F_{1}(\tau), \tau\right)
$$

transforms equation (5.23) into

$$
\partial_{\tau} \tilde{g}+\partial_{z} \tilde{g}\left(1+\mu \tilde{G}^{\ell}\right)=-Q_{1}-\tilde{G}^{\ell}
$$

with $\tilde{G}^{\ell}(u, \tau)=G^{\ell}\left(u+\mu F_{1}(\tau), \tau\right)-Q_{1}(\tau)$. We note that, by Lemma 5.3, $G^{\ell}-Q_{1} \in \mathcal{Y}_{\gamma / 8, \tilde{\rho}_{0} / 8, b}^{1}$, with $\tilde{\rho}_{0}=\max \left\{8 \rho_{0}, 8 \mu_{0} C_{\gamma / 8}^{-1}\left\|F_{1}\right\|_{0, b}\right\}$. Therefore, by $\left.i v\right)$ of Lemma 5.2, $\tilde{G}^{\ell} \in \mathcal{Y}_{\gamma / 4, \tilde{\rho}_{0} / 4, b}^{1}$. We take $\tilde{g}=-F_{1}+\bar{g}$ and we notice that $\bar{g}$ has to satisfy the equation

$$
\partial_{\tau} \bar{g}+\partial_{z} \bar{g}\left(1+\mu \tilde{G}^{\ell}\right)=-\tilde{G}^{\ell} .
$$

This equation is under the hypotheses of Lemma 5.8. Indeed, we have already seen that $\tilde{G}^{\ell} \in \mathcal{Y}_{\gamma / 4, \tilde{\rho}_{0} / 4, b}^{1}$. Moreover by Lemma 5.5, $\mathcal{G}\left(\tilde{G}^{\ell}\right) \in \mathcal{Y}_{\gamma / 4, \tilde{\rho}_{0} / 4, b}^{0}$. Hence Lemma 5.8 works in this case with $H=\tilde{G}^{\ell}, \eta=1$ and $\nu=0$.

Let $\bar{g}$ be the solution of equation (5.25) given by Lemma 5.8. We have that $\bar{g} \in \mathcal{Y}_{\gamma / 2, \rho_{2}, b}^{0}$ and $\partial_{u} \bar{g} \in \mathcal{Y}_{\gamma / 2, \rho_{2}, b}^{1}$. Going back to the original variables $(z, \tau)$, it is clear that

$$
g(z, \tau)=-F_{1}(\tau)+\bar{g}\left(z-\mu F_{1}(\tau), \tau\right)
$$

is a solution of equation (5.23). Moreover, since by Lemma $5.8 \bar{g} \in \mathcal{Y}_{\gamma / 2, \rho_{2}, b}^{0}$ and $\partial_{u} \bar{g} \in \mathcal{Y}_{\gamma / 2, \rho_{2}, b}^{1}$, applying $v$ ) from Lemma 5.2, we have that $g \in \mathcal{Y}_{\gamma, \tilde{\rho}_{1}, b}^{0}$ with $\tilde{\rho}_{1}=\max \left\{2 \rho_{2}, 2 \mu_{0} C_{\gamma / 2}^{-1}\left\|F_{1}\right\|_{0, b}\right\}$. We also have that $\partial_{z} g \in \mathcal{Y}_{\gamma, \tilde{\rho}_{1}, b}^{1}$. This is due to the fact that $\partial_{z} g(z, \tau)=\partial_{u} \bar{g}\left(u-\mu F_{1}(\tau), \tau\right)$ and hence we are allowed to apply $\left.i v\right)$ of Lemma 5.2 to $\partial_{u} \bar{g} \in \mathcal{Y}_{\gamma / 2, \rho_{2}, b}^{1} \subset \mathcal{Y}_{\gamma / 2, \tilde{\rho}_{1} / 2, b}^{1}$.

We have proved that equation (5.22) has a solution $\varphi_{0}$ of the form $\varphi_{0}(z, \tau)=$ $z-\tau+\mu g(z, \tau)$ with $g$ satisfying at least that $g \in \mathcal{Y}_{\gamma, \tilde{\rho}_{1}, b}^{0}$ and $\partial_{z} g \in \mathcal{Y}_{\gamma, \tilde{\rho}_{1}, b}^{1}$. Now we check that $\psi_{0}(z, \tau)=\left(\varphi_{0}(z, \tau), \tau\right)$ is injective in $E_{\gamma, \rho_{1}} \times S_{b}$ if $\rho_{1}$ is big enough. Indeed, let $\left(z_{1}, \tau_{1}\right),\left(z_{2}, \tau_{2}\right) \in E_{\gamma, \rho} \times S_{b}$ be such that $\psi_{0}\left(z_{1}, \tau_{1}\right)=\psi_{0}\left(z_{2}, \tau_{2}\right)$. Clearly $\tau_{1}=\tau_{2}$. Assume that $z_{1} \neq z_{2}$. Then by the mean's value theorem,

$$
\left|z_{1}-z_{2}\right| \leq|\mu||| \partial_{z} g \|_{1, b}\left|z_{1}-z_{2}\right| \rho_{1}^{-1}<\left|z_{1}-z_{2}\right|
$$

if $\rho_{1} \geq \max \left\{\tilde{\rho}_{1}, 2 \mu_{0}\left\|\partial_{z} g\right\|_{1, b}\right\}$, which is a contradiction. 
5.4.1. Proof of Lemma 5.8 To prove Lemma 5.8 we will find an explicit solution of equation (5.24) by means of a suitable linear operator.

We fix $\gamma, b, \rho>0$ and $\nu, \eta, H$ satisfying the hypotheses of Lemma 5.8 and we define the linear operator

$$
\mathcal{F}(f)=-\partial_{z} \mathcal{G}(\mu H \cdot f)
$$

Lemma 5.9 There exists $\rho_{2}=\rho_{2}(\gamma, b, \nu, \eta, \rho) \geq 2 \rho$ such that the operator $(\operatorname{Id}-\mathcal{F})$ is invertible in $\mathcal{Y}_{\gamma, \rho_{2}, b}^{\nu+1}$.

Proof. Let $\rho_{2}=\max \left\{2 \rho,\left(2 \mu_{0} C_{\nu+1, \gamma}\|H\|_{\mathcal{Y}_{\gamma, \rho, b}^{\eta}}\right)^{1 / \eta}\right\}$ where $C_{\nu+1, \gamma}$ is the constant defined in Lemma 5.5. We denote $\mathcal{Y}_{\gamma, \rho_{2}, b}^{l}$ and $\|\cdot\|_{\mathcal{Y}_{\gamma, \rho_{2}, b}^{l}}^{l}$ simply by $\mathcal{Y}^{l}$ and $\|\cdot\|_{l, b}$ respectively.

Since $\mathcal{F}$ is a linear map we only need to check that $\|\mathcal{F}\|_{\nu+1, b}<1$. Let $f \in \mathcal{Y}^{\nu+1}$. We have that $H \in \mathcal{Y}_{\gamma, \rho, b}^{\eta} \subset \mathcal{Y}^{\eta}$, hence by $\left.i i\right)$ of Lemma 5.2, we deduce that $H \cdot f \in$ $\mathcal{Y}^{\nu+\eta+1} \subset \mathcal{Y}^{\nu+1}$ provided $\eta>0$. Moreover, $\|H \cdot f\|_{\nu+1, b} \leq \rho_{2}^{-\eta}\|H\|_{\eta, b}\|f\|_{\nu+1, b}$. Therefore, by Lemma 5.5 and using definition (5.26) of $\mathcal{F}$, we have that

$\|\mathcal{F}(f)\|_{\nu+1, b} \leq \mu_{0} C_{\nu+1,2 \gamma}\|H \cdot f\|_{\nu+1, b} \leq \mu_{0} C_{\nu+1,2 \gamma} \rho_{2}^{-\eta}\|H\|_{\eta, b}\|f\|_{\nu+1, b}<\frac{1}{2}\|f\|_{\nu+1, b}$

provided that $\|H\|_{\eta, b} \leq\|H\|_{\mathcal{Y}_{\gamma, \rho, b}^{\eta}}$ and $\rho_{2}^{\eta} \geq 2 \mu_{0} C_{\nu+1, \gamma}\|H\|_{\mathcal{Y}_{\gamma, \rho, b}^{\eta}}$.

We claim that if either $\nu>0$ or, $\nu=0$ and $\eta \geq 1$,

$$
\partial_{z} \mathcal{G}(H) \in \mathcal{Y}_{\gamma, \rho_{2}, b}^{\nu+1}
$$

with $\rho_{2}$ defined in Lemma 5.9. Indeed, first we deal with the case $\nu>0$. By hypothesis $\mathcal{G}(H) \in \mathcal{Y}_{\gamma / 2, \rho, b}^{\nu}$, therefore using iii) of Lemma $5.2, \partial_{z} \mathcal{G}(H) \in \mathcal{Y}_{\gamma, 2 \rho, b}^{\nu+1} \subset \mathcal{Y}_{\gamma, \rho_{2}, b}^{\nu+1}$ provided that $\nu>0$. In the case $\nu=0$ and $\eta \geq 1$, we recall that $H \in \mathcal{Y}_{\gamma / 2, \rho, b}^{\eta}$. Thus, using Lemma 5.5 we conclude that $\partial_{z} \mathcal{G}(H) \in \mathcal{Y}_{\gamma / 2, \rho, b}^{\eta}$. The claim is proved in this case taking into account that $\eta \geq 1$.

Now we define the functions

$$
\bar{h}=(\operatorname{Id}-\mathcal{F})^{-1}\left(-\partial_{z} \mathcal{G}(H)\right)
$$

and

$$
h=-\mathcal{G}(H)-\mathcal{G}(\mu H \cdot \bar{h}) .
$$

We notice that, by (5.27) and Lemma $5.9, \bar{h} \in \mathcal{Y}_{\gamma, \rho_{2}, b}^{\nu+1}$.

It only remains to check that $h$ so constructed is a solution of equation (5.24). First we note that, since $\mathcal{F}(\bar{h})=\bar{h}+\partial_{z} \mathcal{G}(H)$, we have that

$$
\partial_{z} h=-\partial_{z} \mathcal{G}(H)-\partial_{z} \mathcal{G}(\mu H \cdot \bar{h})=-\partial_{z} \mathcal{G}(H)+\mathcal{F}(\bar{h})=\bar{h} .
$$

Therefore $\partial_{z} h \in \mathcal{Y}_{\gamma, \rho_{2}, b}^{\nu+1}$. Moreover, substituting $\bar{h}$ by $\partial_{z} h$ in (5.28) we obtain that

$$
h=-\mathcal{G}(H)-\mathcal{G}\left(\mu H \cdot \partial_{z} h\right) .
$$

Consequently $h$ is a solution of equation (5.24). Finally, using that $H \in \mathcal{Y}_{\gamma / 2, \rho, b}^{\eta}$, that $\mathcal{G}(H) \in \mathcal{Y}_{\gamma / 2, \rho, b}^{\nu}$ and Lemma 5.5, we conclude that $h \in \mathcal{Y}_{\gamma / 2, \rho, b}^{\nu} \cap \mathcal{Y}_{\gamma, \rho_{2}, b}^{\nu+\eta} \subset \mathcal{Y}_{\gamma, \rho_{2}, b}^{\nu}$ and the lemma is proved. 


\subsection{End of the proof of Theorem 3.3}

We fix $\gamma>0,0<b<b_{0}$. We write $\varphi_{0}(z, \tau)=z-\tau+\mu g(z, \tau)$ where $g$ is the function that satisfies the conclusions of Proposition 5.7. As we claimed in the previous subsection this implies that $\varphi_{0}$ is a solution of equation (5.7). Moreover $\psi_{0}(z, \tau)=\left(\varphi_{0}(z, \tau), \tau\right)$ is injective on $E_{\gamma, \rho_{1}}$ where $\rho_{1}$ is given by Proposition 5.7.

Therefore as we pointed out at the beginning of this Section, any solution of equation (5.7) can be expressed as a function of $\varphi_{0}$. In particular, there exists an analytic function $\chi$ such that

$$
\Delta \phi_{1}=\chi\left(\varphi_{0}\right) .
$$

5.5.1. Proof of the asymptotic expression (3.5) of Theorem 3.3 We claim that $\partial_{\zeta} \chi(\zeta)$ goes to 0 as $\operatorname{Im} \zeta \rightarrow-\infty$. Indeed, we notice that, if $z \in E_{\gamma, \rho_{1}}$ with $|\operatorname{Im} z|$ big enough, $|\operatorname{Re} z|<-\gamma^{-1} \operatorname{Im} z$ and $|\operatorname{Im} \tau|<b \leq-\operatorname{Im} z / 3$. Then, since $g \in \mathcal{Y}_{\gamma, \rho_{1}, b}^{\ell-2 r}$, we have that $|\operatorname{Im} \mu g(z, \tau)| \leq-\operatorname{Im} z / 3$, if $|\operatorname{Im} z|$ is big enough, and thus

$$
5 \operatorname{Im} z / 3 \leq \operatorname{Im}(z-\tau+\mu g(z, \tau)) \leq \operatorname{Im} z / 3
$$

Moreover, from the fact that $\partial_{z} \Delta \phi_{1}(z, \tau)$ goes to 0 as $\operatorname{Im} z \rightarrow-\infty$ :

$$
\begin{aligned}
\lim _{\operatorname{Im} \zeta \rightarrow-\infty} \partial_{\zeta} \chi(\zeta) & =\lim _{\operatorname{Im} z \rightarrow-\infty} \partial_{\zeta} \chi(z-\tau+\mu g(z, \tau)) \\
& =\lim _{\operatorname{Im} \rightarrow-\infty} \partial_{z} \Delta \phi_{1}(z, \tau)\left(1+\mu \partial_{z} g(z, \tau)\right)^{-1}=0 .
\end{aligned}
$$

In the last equality we have used that $\partial_{z} g \in \mathcal{Y}_{\gamma, \rho_{1}, b}^{\ell-2 r+1}$ and that $\Delta \phi_{1}=\chi\left(\varphi_{0}\right)$.

On the other hand, since $\Delta \phi_{1}$ and $g$ are $2 \pi$-periodic with respect to $\tau$,

$\chi(z-\tau+\mu g(z, \tau))=\chi(z-\tau-2 \pi+\mu g(z, \tau+2 \pi))=\chi(z-\tau-2 \pi+\mu g(z, \tau))$

which implies that $\chi$ is $2 \pi$-periodic. Hence, $\partial_{\zeta} \chi$ can be expressed as a Fourier series of the form

$$
\partial_{\zeta} \chi(\zeta)=\sum_{k \in \mathbb{Z}} \mathrm{i} k \chi_{k}(\mu) \mathrm{e}^{\mathrm{i} k \zeta}
$$

where $\left\{\chi_{k}\right\}_{k \in \mathbb{Z}}$ are analytic functions in $B\left(\mu_{0}\right)$.

Finally the property $\partial_{\zeta} \chi(\zeta) \rightarrow 0$ as $\operatorname{Im} \zeta \rightarrow-\infty$ implies that $\chi_{k}(\mu) \mathrm{e}^{\mathrm{i} k \zeta}$ goes to 0 as $\operatorname{Im} \zeta \rightarrow-\infty$ and hence $\chi_{k}(\mu)=0$ for $k>0$. Then, since $\partial_{z} g \in \mathcal{Y}_{\gamma, \rho_{1}, b}^{1}$ at least, we have that

$$
\begin{aligned}
\partial_{z} \Delta \phi_{1}(z, \tau) & =\sum_{k<0} \mathrm{i} k \chi_{k}(\mu) \mathrm{e}^{\mathrm{i} k(z-\tau+\mu g(z, \tau))}\left(1+\mu \partial_{z} g(z, \tau)\right) \\
& \sim-\mathrm{i} \chi_{-1}(\mu) \mathrm{e}^{-\mathrm{i}(z-\tau+\mu g(z, \tau))} \quad \text { as } \operatorname{Im} z \rightarrow-\infty
\end{aligned}
$$

This gives (3.5) taking $C(\mu)=\chi_{-1}(\mu)$. 
5.5.2. The asymptotic expression (3.6) for $C(0)$ Since $\partial_{z} \phi_{1}^{ \pm}$satisfy equation (4.16) we have that

$$
\left[\partial_{\tau}\left(\partial_{z} \phi_{1}^{ \pm}\right)-\partial_{z}\left(\partial_{z} \phi_{1}^{ \pm}\right)\right](z, \tau)=\ell Q_{0}(\tau) z^{-\ell-1}+O(\mu)
$$

and therefore, from the fact that $\partial_{z} \phi_{1}^{ \pm}$are the unique solutions of (4.16) belonging to $\mathcal{X}_{\gamma, \rho_{1}, b}^{\ell+1, \pm}$ respectively, we obtain that

$$
\partial_{z}\left(\phi_{1}^{-}-\phi_{1}^{+}\right)(z, \tau)=\ell \int_{-\infty}^{+\infty} \frac{Q_{0}(\tau+t)}{(z+t)^{\ell+1}} \mathrm{~d} t+O(\mu)
$$

Finally (3.6) follows from the asymptotic expression (5.31) and the fact that $\partial_{z}\left(\phi^{-}-\right.$ $\left.\phi^{+}\right)=\mu \partial_{z}\left(\phi_{1}^{-}-\phi_{1}^{+}\right)$.

\section{Proof of Corollary 3.5}

First we state a technical lemma.

Lemma 6.1 Let $k \in \mathbb{Z}^{+} \backslash\{0\}$. For any $\gamma>0$, $\rho$ big enough and $z \in E_{\gamma, \rho}$,

$$
\int_{-\infty}^{+\infty} \frac{\mathrm{e}^{\mathrm{i} k t}}{(z+t)^{\ell+1}} \mathrm{~d} t=\mathrm{i}^{\ell+1}|k|^{\ell} \frac{2 \pi}{\Gamma(\ell+1)} \mathrm{e}^{-\mathrm{i} k z}\left(1+O\left(|\operatorname{Im} z|^{-1}\right)\right),
$$

where $\Gamma$ is the Gamma function.

Moreover, if $k<0$,

$$
\left|\int_{-\infty}^{+\infty} \frac{\mathrm{e}^{\mathrm{i} k t}}{(z+t)^{\ell+1}} \mathrm{~d} t\right| \leq 2 K_{\ell+1, \gamma} \mathrm{e}^{-2|k||\operatorname{Im} z|} \frac{1}{|z|^{\ell}} \quad \text { for } z \in E_{\gamma, \rho}
$$

where $K_{\ell+1, \gamma}$ is the constant defined in Lemma 4.6.

Now we prove Corollary 3.5. Substituting the definitions of $a^{k}$ in expression (3.6) and using the asymptotic expressions of the integrals in Lemma 6.1 we get

$$
-\mathrm{i} C(0) \mathrm{e}^{-\mathrm{i}(z-\tau)} \sim \ell \mathrm{i}^{\ell+1} \frac{2 \pi}{\Gamma(\ell+1)} \sum_{k>0} a^{k}|k|^{\ell} \mathrm{e}^{-i k(z-\tau)}\left(1+O\left(|\operatorname{Im} z|^{-1}\right)\right) \text { as } \operatorname{Im} z \rightarrow-\infty .
$$

Therefore, since $a^{1} \neq 0$,

$$
C(0)=-\mathrm{i}^{\ell} \frac{2 \pi \ell}{\Gamma(\ell+1)} a^{1} \neq 0 .
$$

Finally $i$ ) from Corollary 3.5 follows from the asymptotic expression (3.5), (6.2) and the fact that $g$ goes to 0 as $\operatorname{Im} z \rightarrow-\infty$ and $i i$ ) is proved from (3.5) and (6.2).

Proof of Lemma 6.1. Let $z \in E_{\gamma, \rho}$. First we deal with $k<0$. By Cauchy's theorem we can move the path of integration obtaining

$$
\int_{-\infty}^{+\infty} \frac{\mathrm{e}^{\mathrm{i} k t}}{(z+t)^{\ell+1}} \mathrm{~d} t=\mathrm{e}^{-2 k \operatorname{Im} z} \int_{-\infty}^{+\infty} \frac{\mathrm{e}^{\mathrm{i} k t}}{(z+t+2 \mathrm{i} \operatorname{Im} z)^{\ell+1}} \mathrm{~d} t .
$$

Let $\tilde{z}=z+2 \mathrm{i} \operatorname{Im} z$. We note that $\tilde{z},-\tilde{z} \in D_{\gamma, \rho}^{+} \cap D_{\gamma, \rho}^{-}$and $|\tilde{z}| \geq|z|$. Using bound (4.24) in (6.3) we get the result. 
Now we deal with $k>0$. Performing trivial changes of variables we have that

$$
\int_{-\infty}^{+\infty} \frac{\mathrm{e}^{\mathrm{i} k t}}{(z+t)^{\ell+1}} \mathrm{~d} t=\frac{\mathrm{e}^{-\mathrm{i} k \operatorname{Re} z}}{|\operatorname{Im} z|^{\ell}} \int_{-\infty}^{+\infty} \frac{\mathrm{e}^{\mathrm{i} k t|\operatorname{Im} z|}}{(t-\mathrm{i})^{\ell+1}} \mathrm{~d} t .
$$

In [3] (pp 80, formula (6.28)) the following expression is given:

$$
\int_{-\infty}^{+\infty} \frac{\mathrm{e}^{\mathrm{i} k t / \varepsilon}}{(t-\mathrm{i} c)^{\ell+1}} \mathrm{~d} t=\mathrm{i}^{\ell+1}\left(\frac{k}{\varepsilon}\right)^{\ell} \frac{2 \pi}{\Gamma(\ell+1)} \mathrm{e}^{-k c / \varepsilon}(1+O(\varepsilon))
$$

with $\varepsilon>0, c>0$ and $k>0$. Putting $\varepsilon=|\operatorname{Im} z|^{-1}$ and $c=1$ in (6.5) we get (6.1) from (6.4).

We point out that, if $\ell \in \mathbb{N}$, the asymptotic expressions of this lemma can be easily obtained by using residues theory

\section{Appendix}

In this study, we have restricted ourselves to the case in which our initial Hamiltonian $\mathcal{H}=\mathcal{H}_{0}+\mu \mathcal{H}_{1}$ is analytic with respect to $\tau$, but this hypothesis is, in fact, not necessary. The purpose of this appendix is to justify that our proofs are also valid in a more general setting: the differentiable case with respect to $\tau$.

First we present the precise statement of the results which ensures that, with the obvious changes, Theorems 3.1, 3.3 and Corollary 3.5 are also valid in the differentiable case.

Theorem 6.2 Consider the Hamiltonian system $\mathcal{H}=\mathcal{H}_{0}+\mu \mathcal{H}_{1}$ with

$$
\mathcal{H}_{0}(z, w)=\frac{1}{2} w^{2} z^{2 r}-\frac{1}{2 z^{2 r}}, \quad \mathcal{H}_{1}(z, w, \tau)=\frac{1}{z^{\ell}} \sum_{j=0}^{N} A_{j}(\tau, \mu) z^{2 r j} w^{j}
$$

where $r \geq 1, \ell \in \mathbb{R}, N \in \mathbb{N}$ and $\left\{A_{j}\right\}_{j \in\{0, \cdots, N\}}$ are arbitrary $2 \pi$-periodic functions with respect to $\tau$, analytic with respect to $\mu$ in $B\left(\mu_{0}\right)$, for some $\mu_{0}>0, C^{q}$ with respect to $\tau$ and such that the Fourier series of $A_{j}$ is uniformly convergent for all $j \in\{0, \cdots, N\}$.

Then, if $\ell \geq 2 r$, for all $\gamma>0$ there exists $\rho_{0}=\rho_{0}(\gamma, q, \ell, r)>0$ such that the Hamilton-Jacobi equation associated to $\mathcal{H}$ has solutions $\phi^{ \pm}: \mathcal{D}_{\gamma, \rho_{0}, b}^{ \pm} \rightarrow \mathbb{C}$ of the form $\phi^{ \pm}=\phi_{0}+\mu \phi_{1}^{ \pm}, C^{q+1}$ and $2 \pi$-periodic with respect to $\tau$, and analytic with respect to $(z, \mu)$. Moreover $\partial_{z} \phi_{1}^{ \pm}$is determined by the condition

$$
\sup _{(z, \tau, \mu) \in \mathcal{D}_{\gamma, \rho_{0}, b}^{ \pm}}\left|z^{\ell+1} \partial_{z} \phi_{1}^{ \pm}(z, \tau, \mu)\right|<+\infty .
$$

Theorem 3.3 and Corollary 3.5 are also true in this new setting taking into account the new regularity of $g$ with respect to $\tau$, that is: $g$ is $C^{q}$, analytic with respect to $(z, \mu) \in E_{\gamma, \rho} \times B\left(\mu_{0}\right)$ and such that the Fourier series of $g$ is uniformly convergent.

To justify this result we take advantage from the fact that our results are valid for spaces of Fourier series satisfying the properties given in Subsection 4.1 (and consequently in Subsection 5.1). 
The appropriate Banach spaces in this case are defined as follows. Let $\gamma, \rho>0$ and $\nu \in \mathbb{R}$. We define the space $\mathcal{Z}_{\gamma, \rho}^{\nu, \pm}$ of Fourier series $f(z, \tau, \mu)=\sum_{k \in \mathbb{Z}} f_{k}(z, \mu) \mathrm{e}^{\mathrm{i} k \tau}$, with $f_{k} \in X_{ \pm}^{\nu}$ analytic with respect to $(z, \tau) \in D_{\gamma, \rho}^{ \pm} \times B\left(\mu_{0}\right), C^{0}$ and such that the Fourier series of $f$ is uniformly convergent. We endow $\mathcal{Z}_{\gamma, \rho}^{\nu, \pm}$ with the norm

$$
\|f\|_{\nu, 0}=\sum_{k \in \mathbb{Z}}\left\|f_{k}\right\|_{\nu}
$$

and it becomes a Banach space. This fact can be proved as in [23].

It is straightforward to check that the Banach spaces $\mathcal{Z}_{\gamma, \rho}^{\nu, \pm}$ satisfy the properties given in Lemma 4.3 and Lemma 4.4. (We only need to take $b=0$ and replace analyticity with respect to $\tau$ by continuity).

Without any change in the procedure given in Section 4 we can check that there exists a solution, $\varphi^{+} \in \mathcal{Z}_{\gamma, \rho}^{\ell+1,+}$ of the fixed point equation $\varphi^{+}=\mathcal{B}\left(\psi_{1}^{\ell}+\partial_{z} \psi_{2}^{\ell}\left(\varphi^{+}\right)\right)$and hence $\varphi^{+}$is $C^{1}$ with respect to $\tau$ since $\partial_{\tau} \varphi^{+}=\psi_{1}^{\ell}+\partial_{z} \psi_{2}^{\ell}\left(\varphi^{+}\right)-\partial_{z}\left(\mathcal{B}\left(\psi_{1}^{\ell}+\partial_{z} \psi_{2}^{\ell}\left(\varphi^{+}\right)\right)\right.$. If either $\ell>2 r$, or $\ell=2 r$ with $Q_{1}=0$ and $\left\langle F_{0} \cdot Q_{2}\right\rangle=0$, we have that $\partial_{z} \phi_{1}^{+}=\varphi^{+}$ and therefore $\partial_{z} \phi_{1}^{+}$is differentiable with respect to $\tau$. Moreover, using definition (4.33) of $\phi_{1}^{+}$we conclude that $\phi_{1}^{+}$is differentiable with respect to $\tau$, differentiating under the integral sign. In the especial case $\ell=2 r$ and either $Q_{1} \neq 0$ or $\left\langle F_{0} \cdot Q_{2}\right\rangle \neq 0$, we have that $\partial_{z} \phi_{1}^{+}(z, \tau)=\varphi^{+}\left(z-\mu F_{1}(\tau), \tau\right)$. Hence $\varphi^{+}$is $C^{1}$ with respect to $\tau$ and henceforth, we have the same property for $\partial_{z} \phi_{1}^{+}$and $\phi_{1}^{+}$. We deal with the - case in an analogous way.

Therefore we conclude that there exist solutions $\phi^{ \pm}=\phi_{0}+\mu \phi_{1}^{ \pm}$of the HamiltonJacobi equation $\partial_{\tau} \phi^{ \pm}+\mathcal{H}\left(z, \partial_{z} \phi^{ \pm}, \tau\right)$ of the form stated in Theorem 6.2 and satisfying that they are $C^{0}$, that their Fourier series is uniformly convergent.

Finally we observe that, since $\phi^{ \pm}=\phi_{0}+\phi_{1}^{ \pm}$with $\partial_{z} \phi_{1}^{ \pm} \in \mathcal{Z}_{\gamma, \rho}^{\ell+1, \pm}$, then $\partial_{z} \phi^{ \pm} \in \mathcal{Z}_{\gamma, \rho}^{2 r, \pm}$. In particular we have that $\partial_{z} \phi^{ \pm}$is $C^{0}$ and the Fourier series of $\phi^{ \pm}$are uniformly convergent. On the one hand, we notice that since $\phi^{ \pm}$are analytic with respect to $z, \partial_{z} \phi^{ \pm}$and consequently $\mathcal{H}\left(z, \partial_{z} \phi^{ \pm}, \tau\right)$ are $C^{0}$ (here we have used that $\mathcal{H}$ is $C^{q}$ ). On the other hand, since $\phi^{ \pm}$is a solution of the Hamilton-Jacobi equation associated to $\mathcal{H}$, $\partial_{\tau} \phi^{ \pm}=-\mathcal{H}\left(z, \partial_{z} \phi^{ \pm}, \tau\right)$ and thus $\phi^{ \pm}$is $C^{1}$. An inductive argument allows us to conclude that $\phi^{ \pm}$is $C^{q+1}$.

For the second part of Theorem 6.2, we follow the same steps as in Section 5. We omit the details of the proof because they are quite analogous. Section 6 works without any change.

\section{Acknowledgments}

I wish to thank the Institut de Mécanique Celeste for their hospitality and especially David Sauzin for extremely useful discussions. I am also indebted to E. Fontich and T.M. Seara for valuable remarks.

The study received the partial support of the Catalan Grant CIRIT 2001SGR-70 and the Spanish Grant DGICYT BFM2003-09504-C02-01. 


\section{References}

[1] Angenent S 1993 A variational interpretation of Melnikov's function and exponentially small separatrix splitting Symplectic geometry, London Math. Soc. Lecture Note Ser., vol. 192, Cambridge Univ. Press, pp. 5-35.

[2] V.I. Arnold 1964 Instability of dynamical systems with many degrees of freedom Soviet Math. Dokl. 5 581-585.

[3] Baldomá I and Fontich E 2004 Exponentially small splitting of invariant manifolds of parabolic points Memoirs of AMS vol. 167 (792) p x+83.

[4] Bonet C Sauzin D Seara TM and València M 1998 Adiabatic invariant of the harmonic oscillator, complex matching and resurgence methods SIAM J. Math. Anal. 29 (6) 1335-1360.

[5] Candelpergher B Nosmas JC and Pham F 1993 Approche de la résurgence Actualités Math. Hermann Paris.

[6] Delshams A and Ramírez-Ros R 1998 Exponentially small splitting of separatrices for perturbed integrable standard-like maps J. Nonlinear Sci. 8 317-352.

[7] Delshams A and Seara TM 1992 An asymptotic expression for the splitting of separatrices of the rapidly forced pendulum Comm. Math. Phys. 150 433-463.

[8] Delshams A and Seara TM 1997 Splitting of separatrices in Hamiltonian systems with one and a half degrees of freedom Math. Phys. Electron. J. 3 4-40.

[9] Écalle J 1981, 1985 Les fonctions résurgentes Publ. Math. d'Orsay Vol. 1: 81-05 Vol. 2: 81-06, Vol. 3:85-05 Paris.

[10] Fontich F 1993 Exponentially small upper bounds for the splitting of separatrices for high frequency periodic perturbations Nonlinear Anal. 20 733-744.

[11] Fontich F 1995 Rapidly forced planar vector fields and splitting of separatrices J. Differential Equations 119 310-335.

[12] Fontich F and Simó C 1990 The splitting of separatrices for analytic diffeomorphisms Ergodic Theory Dynam. Systems 10 295-318.

[13] Gelfreich VG 1997 Melnikov method and exponentially small splitting of separatrices Phys. D 101 227-248.

[14] Gelfreich VG 1999 A proof of the exponentially small transversality of the separatrices for the standard map Comm. Math. Phys. 201 (1) 155-216.

[15] Gelfreich VG and Sauzin D 2001 Borel summation and splitting of separatrices for the Hénon map Ann. Inst. Fourier, Grenoble 51 (2) 1001-1055.

[16] Holmes P Marsden J and Scheurle J 1988 Exponentially small splitting of separatrices with applications to KAM theory and degenerate bifurcations Contemp. Math. vol. 81 213-244.

[17] Lazutkin VF 1984 Splitting of separatrices for the Chirikov's standard map VINITI 6372/84. (English version in www.maia.ub.es/mp_arc \#98-421.)

[18] Melnikov VF 1963 On the stability of the center for time periodic perturbations Trans. Moscow Math. Soc. 12 3-56.

[19] Olivé C and Seara TM 1999 Matching complejo y resurgencia en el problema de la escisión de separatrices Actas CEDYA99 419-426.

[20] Olivé C Sauzin D and Seara TM 2003 Resurgence in a Hamilton-Jacobi equation Ann. Inst. Fourier, Grenoble 53 (4) 1185-1235.

[21] Poincaré H 1890 Sur le problème des trois corps et les équations de la dynamique Acta Math. 13 $1-271$.

[22] Sauzin D 1995 Résurgence paramétrique et exponentielle petitesse de l'écart des séparatrices du pendule rapidement forcé Ann. Inst. Fourier, Grenoble 45 (2) 453-511.

[23] Sauzin D 2001 A new method for measuring the splitting of invariant manifolds Ann. Scient. Éc. Norm. Sup. 4 série t. 34 p 159-221.

[24] Scheurle J, Marsden J and Holmes P 1991 Exponentially small estimates for separatrix splitting Asymptotics beyond all orders (La Jolla, CA, 1991) NATO Adv. Sci. Inst. Ser. B Phys. vol. 284 
Inner equation for $1 \frac{1}{2}$ degrees of freedom Hamiltonian systems

Plenum, New York, 187-195.

[25] Treschev DV 1997 Separatrix splitting for a pendulum with rapidly oscillating suspension point, Russ. J. Math. Phys. 5 (2) 63-98. 